\title{
Balkanologie
}

Balkanologie Revue d'études pluridisciplinaires

Vol. XI, $n^{\circ} 1-2$ | 2008

Volume XI Numéro 1-2

\section{Imaginaires et itinéraires migratoires bulgares en Europe. Une introduction}

\section{Nadège Ragaru}

\section{OpenEdition}

\section{Journals}

Édition électronique

URL : http://journals.openedition.org/balkanologie/873

DOI : $10.4000 /$ balkanologie. 873

ISSN : 1965-0582

Éditeur

Association française d'études sur les Balkans (Afebalk)

\section{Référence électronique}

Nadège Ragaru, «Imaginaires et itinéraires migratoires bulgares en Europe. Une introduction », Balkanologie [En ligne], Vol. XI, n 1-2 | 2008, mis en ligne le 31 décembre 2008, consulté le 17 décembre 2020. URL : http://journals.openedition.org/balkanologie/873 ; DOI : https://doi.org/ 10.4000/balkanologie.873

Ce document a été généré automatiquement le 17 décembre 2020.

(C) Tous droits réservés 


\title{
Imaginaires et itinéraires migratoires bulgares en Europe. Une introduction
}

\author{
Nadège Ragaru
}

1 En 2003, Dana Diminescu ouvrait sa réflexion sur les mobilités roumaines en Europe avec le constat d'un décalage entre le nombre des migrants et leur visibilité dans les espaces publics des pays de destination ${ }^{1}$. La surexposition médiatique des citoyens roumains en mouvement allait de pair avec la construction de représentations sociales négatives associées à des images de criminalité et d'illégalité. Les parcours des migrants originaires de Bulgarie suggèrent de prime abord l'observation inverse. Jusque récemment, ni la Bulgarie, ni les Bulgares n'avaient, à tout le moins en France, attiré l'attention des media ou des observateurs politiques. Mobilités il y avait assurément, mais de cette thématique-là il n'était guère question, y compris dans les travaux universitaires. Ce silence s'explique partiellement au regard du développement modeste des études bulgares en France. Dans le domaine des migrations, le poids démographique de la Bulgarie (7,9 millions d'habitants selon le recensement de 2001) a en outre convaincu les responsables d'organisations internationales et d'agences gouvernementales - commanditaires fréquents des recherches empiriques - que cet Etat ne présentait qu'un potentiel migratoire limité et constituait dès lors un site d'observation secondaire ${ }^{2}$.

2 La faible couverture des mobilités bulgares ne constitue toutefois pas l'apanage des écrits d'experts ou des travaux académiques en langue française ou anglaise. La thématique a également tardé à susciter l'intérêt des sciences sociales bulgares ${ }^{3}$. Ce, alors que les circulations migratoires - qui ne mobilisaient pas exclusivement le répertoire de l'ethnicité «turque » ou «rom»- ont été amorcées dès la chute du communisme et qu'elles ont rapidement contribué au développement de formes de sociabilité et d'arts de faire partiellement inédits, au refaçonnage des imaginaires sociaux du statut et des hiérarchies, à la transformation des rapports au temps et à l'espace. Dans les media, dans les conversations ordinaires, jusque vers la fin des années 
1990 ces horizons du départ sont principalement thématisés sur le mode de la déperdition (" fuite des cerveaux », exode des jeunes) et de la déploration devant ce qui est vécu comme une inexorable dislocation des tissus sociaux et une lente érosion de la "bulgarité » même $e^{4}$. Nombreux sont les récits de familles déchirées par les déplacements incessants de pères et mères qui confient leurs enfants à des proches tout en espérant que les effets de ces séparations seront compensés par la plus grande aisance matérielle offerte aux enfants. Récurrentes sont les évocations de villages désertés, abandonnés à des personnes âgées sans avenir...ou à quelques retraités occidentaux, britanniques notamment, que séduit une "authenticité rurale " dans laquelle ils peinent à lire les traces du désarroi ${ }^{5}$.

3 Au tournant du siècle, cependant, les évocations des migrations commencent à se diversifier. Les premières rubriques consacrées aux "émigrants" (emigranti), aux "Bulgares à l'étranger» (bălgarite v čužbina) font leur apparition dans la presse quotidienne et hebdomadaire. Aux dénonciations douloureuses de la stratification (sociale, générationnelle, de genre) magnifiée par les installations dans la mobilité se conjugue désormais une célébration de la réussite des Bulgares partis ailleurs (leurs qualités, leurs performances, la reconnaissance acquise "là-bas»). Le détour par l'international, parfois encore soupçonné d'alimenter un brain drain, est aussi progressivement érigé en jalon indispensable à tout parcours de réussite sociale. Dans les classes supérieures, maints parents envoient leurs enfants à l'étranger dans des établissements privés, onéreux et réputés dès le lycée. L'âge du départ (avant ou après l'entrée à l'université ?) devient à lui seul un marqueur social ${ }^{6}$. Les transferts monétaires, la distribution de cadeaux et les pratiques d'évergétisme social contribuent à transformer les paysages ruraux et urbains. Y compris chez ceux qui restent, l'horizon du départ s'inscrit comme un repère, voire une norme. A l'instar de la société bulgare dont les images, éclatées, ont revêtu des contrastes grandissants, les figures migrantes en viennent à solliciter une pluralité d'associations - à la prospérité comme à la crise, à la «modernité » comme à une "régression », à l'« ouverture » comme à la dilution de soi. La Bulgarie démultipliée à travers ses citoyens en migration apparaît striée de parcours individuels et collectifs où se dessinent des lieux éphémères de rencontre et de reconnexion, de frottement aussi, dans le silence d'une nuit au Canada, aux Etats-Unis, en France ou ailleurs, où de jeunes diplômés polyglottes, poly-insérés dans la fluidité d'un monde globalisé, déversent parfois un fiel de tristesse et de rancœurs sur des forums de discussions bulgares.

4 Tardivement advenu à la conscience des enjeux politiques et économiques des nouvelles migrations, l'Etat tente à son tour de mobiliser le capital social de Bulgares dont certains ont réussi leur insertion professionnelle à l'international. C'est l'époque où, en avril 2000, le gouvernement Kostov (UFD, droite) lance une initiative connue sous le nom de Velikden (la Pâque) afin de réunir les cadres de la « diaspora bulgare » en Europe et aux Etats-Unis ${ }^{7}$. L'objectif est de valoriser leur potentiel d'intermédiation auprès des investisseurs étrangers, voire d'encourager les «Bulgares de l'étranger » à revenir dans leur pays ${ }^{8}$. Siméon de Saxe-Cobourg-Gotha, le successeur d'Ivan Kostov, puisera d'ailleurs dans ce vivier de jupite (yuppies), fringants cadres bancaires à la City ou ailleurs, au moment de former son gouvernement en 2001. La perspective d'un " retour " apparaît pourtant bien fragile lorsque, au début des années 2000 , les études empiriques consacrées aux migrations commencent à se développer en Bulgarie. 
Certaines recherches sont financées par des organismes internationaux dans la perspective du futur élargissement de l'Union européenne (UE) à l'Est (dont la préparation a été couplée avec le transfert vers les Etats candidats de larges responsabilités dans la gestion des courants migratoires). A l'approche des adhésions, décideurs européens et internationaux s'inquiètent de l'impact de l'ouverture sur les marchés du travail nationaux et le développement de la criminalité organisée. L'objectif semble parfois plus de "juguler» que de comprendre, sinon de comprendre pour mieux contrôler. Pourtant, en Bulgarie et dans plusieurs universités européennes, une communauté de chercheurs se forge peu à peu, désireuse d'aborder dans une démarche non normative - les profils et stratégies des migrants, ainsi que l'effet des nouvelles mobilités sur les définitions de la réussite sociale, les identifications ethniques et religieuses ou encore les constructions de la citoyenneté et du rapport à l'Etat. Le dossier proposé ici en partenariat avec la section balkanique de l'Institut et du Musée d'ethnographie de l'Académie des sciences bulgares (BAN) a vocation à mieux faire connaître ces écrits à un public francophone.

6 Après une brève mise en perspective des mobilités bulgares postérieures à 1989, le regard se déplacera vers les modes de constitution, en Bulgarie, d'un champ de recherche sur les enjeux migratoires, ainsi que vers les questionnements au croisement desquels les faits migratoires ont été saisis. Enfin, l'on présentera le prisme spécifique retenu dans le présent numéro de Balkanologie.

\section{I - Ressources, parcours et temporalités migrantes : retour sur les années 1989-2008}

7 Le développement de circulations bulgares après 1989 intervient dans un triple contexte : la chute du communisme et la formation d'une économie de marché; une "globalisation" affectant, entre autres, l'organisation des économies nationales, les notions de frontières et d'espace et les imaginaires du possible et du pensable; la refonte des contours géographiques et des priorités de l'Union européenne (avec l'accentuation de la dimension sécuritaire des politiques publiques). Au niveau européen, les mouvements de citoyens bulgares participent en outre d'une mutation du système des mobilités corrélée au développement de flux migrants depuis l'Est de l'Europe vers le Sud (Portugal, Espagne, Italie, Grèce), autrefois zone traditionnelle d'émigration. Apprécier ces mises en mouvement impose d'envisager plusieurs espaces-temps : les migrations diffèrent dans leurs modalités, leur durée, le profil des participants, ainsi que leurs finalités en fonction des destinations et des périodes envisagées. En 2005, Ralitza Soultanova distinguait les demandes d'asile du début des années $1990^{10}$, le "commerce de la valise», les mobilités de personnels hautement qualifiés et d'étudiants, les migrations «longue distance» visant une installation durable (Etats-Unis, Canada, Australie, Nouvelle-Zélande, etc.), les migrations irrégulières et les conventions d'échange de travailleurs ${ }^{11}$. Petya Kabakchieva préfère retenir une tripartition - migrants à installation définitive (settlers), migrants temporaires (Gastarbeiter) et migrants saisonniers - qui lui permet d'envisager des rapports distincts à l'espace, aux identités sociales et individuelles, aux rencontres avec d'autres cultures ${ }^{12}$. L'adoption d'un découpage chronologique, aussi imparfait soit-il, aidera à apprécier la pluralité de ces dynamiques et leurs inflexions temporelles. 


\section{1 - Une redécouverte de l'international en situation de crise économique (1989-1993)}

8 Les sources des douanes et de la police des frontières suggèrent qu'environ 250000 personnes auraient franchi les frontières de la Bulgarie entre 1989 et $1992^{13}$; ces données agrègent des mouvements très hétérogènes. Soumis entre 1984 et 1989 à une brutale politique d'assimilation forcée, quelque 320 à 340000 Turcs bulgares ont été contraints à un exode forcé à destination de la Turquie entre mai et août 1989. Avant même la fin de l'année 1989, approximativement 150000 migrants turcs étaient rentrés en Bulgarie ${ }^{14}$. Un second type de mouvements est rendu possible par la suppression des anciens visas de sortie communistes bulgares. La curiosité de l'ailleurs, le désir de découvrir un Occident rêvé, attendu, espéré sont renforcés dans certains milieux par la déception que constitue la victoire des anciens communistes lors des premières élections législatives libres de juin 1990, ainsi que par la récession économique transitionnelle ${ }^{15}$.

Dès 1990, des jeunes, des étudiants, des chercheurs et enseignants, mais aussi des actifs hautement qualifiés (microélectronique, nouvelles technologies) décident de s'établir à l'étranger. Le secteur de la recherche est particulièrement affecté par les restructurations post-communistes, la fermeture d'instituts de recherche publics, la réduction des crédits : entre 1988 et 1993, le nombre des personnels chute de $31 \%$, celui des chercheurs de 18,9\% (soit un peu plus de 6000 personnes) ${ }^{16}$. Environ 600 chercheurs (médecine, chimie, architecture, biologie, physique, géologie, etc.) gagnent alors les Etats-Unis et l'Allemagne, mais aussi l'Afrique, le Canada et la GrandeBretagne. La plupart sont dotés de savoirs linguistiques, d'une expérience antérieure à l'international et/ou d'une insertion dans des espaces de recherches internationalisés ${ }^{17}$. La crise économique et la désorganisation de l'agriculture qu'aggrave la politique de restitution des terres nationalisées par le pouvoir communiste fournissent le contexte dans lequel interviennent de nouveaux départs à compter de 1991. En ces années de dépression, des Turcs, des Roms et des Bulgares musulmans pratiquent le " commerce de la valise », mettant à profit les différentiels de prix et d'accessibilité de biens de consommation (vêtements, alimentation, cigarettes et alcool) entre la Bulgarie et la Turquie, la Pologne et la Bulgarie, la Bulgarie et la Yougoslavie, etc. ${ }^{18}$. Certains Turcs bulgares cherchent à s'installer en Turquie (où ils rejoignent parfois des membres de leur famille dont ils ont été séparés en 1989), mais se heurtent à l'obligation de visa imposée par la Turquie en août 1989 (elle restera en vigueur jusqu'en 2001). Au cours de cette période, maints franchissements de la frontière s'effectuent illégalement grâce à des « passeurs » ${ }^{19}$.

11 Jusqu'au milieu des années 1990, les pays d'Europe centrale (Allemagne, Autriche, à un moindre degré, la République tchèque et la Hongrie) figurent parmi les destinations privilégiées par les migrants bulgares. La nature des procédures d'asile en vigueur jusqu'en 1993, une certaine familiarité entretenue à l'époque communiste avec la RDA, le dynamisme du marché du travail et une réputation de prospérité contribuent à expliquer l'attractivité de l'Allemagne aux yeux des Bulgares, des Turcs bulgares et des Roms bulgares. Dès 1991-1992, la Bulgarie et l'Allemagne concluent par ailleurs des accords encadrant l'envoi de travailleurs temporaires (principalement dans le secteur du tourisme et des services) ${ }^{20}$. Vers le milieu des années 1990, s'observe cependant une diversification des candidats au départ et une inflexion dans leurs itinéraires. Au même 
moment, les politiques migratoires européennes se durcissent avec l'entrée en vigueur de la Convention d'application des Accords de Schengen en 1995 et le placement de certains Etats post-communistes européens sur une «liste noire » des pays soumis à obligation de visa ${ }^{21}$.

\section{2 - La diversification des expériences migrantes (1995-2001)}

\section{(1996-1997), les migrations se poursuivent et s'orientent désormais plus volontiers vers} les pays d'Europe du Sud, au premier chef la Grèce, puis l'Espagne et l'Italie, où des déficits de main d'œuvre sont chroniquement enregistrés dans l'agriculture, le bâtiment, les services à domicile, la restauration, l'hôtellerie et le tourisme ${ }^{22}$. Dans la branche agricole et la construction, les mouvements revêtent un caractère temporaire lié au rythme de travail propres à ces secteurs d'activité : un maillage de circulations migratoires se met alors en place, les séjours à l'étranger alternant avec des retours plus ou moins longs en Bulgarie. Cette tendance aux «migrations maintien» (pour reprendre l'expression de Mirjana Morokvasic) n'est pas propre à la Bulgarie; elle concerne l'ensemble des Etats post-communistes. Les mobilités transnationales y constituent un moyen non de partir définitivement, mais de demeurer chez soi - certes au prix d'absences récurrentes ${ }^{23}$. $\mathrm{du}$ travail européen complexe et contradictoire. En 2000, on estime que près de 4,5 millions de saisonniers (dont 500000 issus de pays extracommunautaires) sont employés dans l'agriculture des Etats membres de l'UE, faisant du recours périodique aux travailleurs étrangers « une caractéristique structurelle de l'agriculture intensive des pays d'Europe $»^{24}$. Une forte croissance économique, le désir de s'assurer une main d'œuvre qualifiée et peu onéreuse conduisent les Etats grec, espagnol et italien à mettre en œuvre des programmes de régularisation de migrants devenus indispensables au fonctionnement de leurs économies (en 1998 et en 2002 pour l'Italie, en 1998 et en 2001 pour la Grèce, en 2000, 2001 et 2005 pour l'Espagne, etc.) $)^{25}$. En contrepartie, la différenciation des statuts et la segmentation des marchés du travail se renforcent, singulièrement dans la branche agricole, le maraîchage ou l'arboriculture. Migrants communautaires, migrants extracommunautaires (mais européens) et migrants originaires d'autres continents sont de plus en plus souvent mis en concurrence ${ }^{26}$.

Du point de vue des citoyens bulgares, le choix de la Grèce, de l'Italie et de l'Espagne renforcé par la constitution progressive de réseaux d'entraide et d'interconnaissance renvoie également à un sentiment de proximité culturelle relative et, dans le cas grec, géographique. En cette seconde moitié des années 1990, cependant, les ressortissants bulgares sont obligés d'obtenir un visa pour se rendre dans les pays de l'espace Schengen. Les conditions d'entrée, de séjour et de travail à l'étranger sont partiellement, voire entièrement irrégulières, et reposent sur l'existence de passeurs et d'intermédiaires. La décision adoptée par le Conseil européen en décembre 2000 de lever les visas Schengen amorce dès lors une troisième période dans l'histoire des migrations bulgares contemporaines. 


\section{3 - Après la levée des visas Schengen (avril 2001)}

15 A partir d'avril 2001, les Bulgares sont autorisés à séjourner moins de trois mois dans les Etats signataires des Accords de Schengen avec des visas de touristes. L'allègement des contraintes juridiques n'est toutefois que partiel puisqu'il n'engage pas un libre accès aux marchés du travail : fréquents sont les dépassements de séjour, les emplois informels non déclarés. Cette nouvelle phase coïncide avec une forte augmentation des départs et confirme l'attractivité croissante des Etats d'Europe du sud (y compris le Portugal, Chypre et Malte). Sur la base d'une enquête quantitative menée après de 1000 foyers en novembre 2005, Veselin Minčev et Venelin Bošnakov distinguent alors deux types de mobilités: les mouvements saisonniers/pendulaires de quelques mois, qui concernent davantage les Etats d'Europe du Sud-Est; des migrations un peu plus longues (de l'ordre d'un an et trois mois en moyenne) à destination de pays du nord de l'Europe, l'Allemagne attirant le plus fort pourcentage de migrants, essentiellement parmi les hommes ${ }^{27}$. Selon leurs estimations, à cette date, $15,2 \%$ de la population bulgare (soit environ 440000 foyers) a d'ores et déjà connu une expérience migratoire, si l'on définit celle-ci comme un séjour à l'étranger d'au moins trois mois ${ }^{28}$.

Dans ce contexte, entre 2001 et 2004 les transferts monétaires que les travailleurs bulgares font parvenir à leurs parents restés en Bulgarie s'accroissent rapidement, fournissant un apport majeur à la balance des paiements courants. Sur la période 2000-2005, le volume annuel des transferts nets courants depuis l'étranger passe de 300 millions environ en 1999-2000 à plus de 900 millions en fin de période (ce qui représente environ $30 \%$ du déficit de la balance des paiements et jusqu'à $4 \%$ du PNB) (voir tableau 1). La Banque centrale bulgare (BNB) estime à 344,7 millions d'euros en 2004 et 335,5 millions d'euros en 2005 la part des versements provenant de Bulgares travaillant à l'étranger ${ }^{29}$. Veselin Minčev et Venelin Bošnakov jugent ces résultats sousestimés et avancent le chiffre de 836 millions d'euros par an en moyenne entre 2001 et $2005\left(1 / 5^{\text {ème }}\right.$ du déficit de la balance des paiements courants et $1 / 3$ des investissements directs étrangers) sur la base de leur enquête auprès des ménages ${ }^{30}$.

Tableau 1 - Flux de transferts courant et privés en Bulgarie

\begin{tabular}{|l|l|l|l|l|l|l|l|}
\hline & $\mathbf{1 9 9 9}$ & $\mathbf{2 0 0 0}$ & $\mathbf{2 0 0 1}$ & $\mathbf{2 0 0 2}$ & $\mathbf{2 0 0 3}$ & $\mathbf{2 0 0 4}$ & $\mathbf{2 0 0 5}$ \\
\hline $\begin{array}{l}\text { Transferts courants, crédits (en millions } \\
\text { d'euros) }\end{array}$ & 309,6 & 386,2 & 674,0 & 676,9 & 762,5 & 1048,4 & 1110,8 \\
\hline Transferts privés, crédits (en millions d'euros) & 232,2 & 295,7 & 450,7 & 517,2 & 600,2 & 798,9 & 720,0 \\
\hline En \% du PNB & 1,9 & 2,2 & 3,0 & 3,1 & 3,4 & 4,1 & 3,4 \\
\hline En \% des exportations & 6,2 & 5,6 & 7,9 & 8,5 & 9,0 & 10,0 & 7,7 \\
\hline En \% des importations & 4,9 & 4,5 & 6,0 & 6,7 & 6,8 & 7,5 & 5,4 \\
\hline En \% de la balance des paiements & 23,0 & 23,1 & 25,3 & 30,6 & 27,3 & 29,3 & 18,1 \\
\hline En \% des IDE & 39,6 & 38,8 & 40,9 & 52,8 & 32,4 & 35,1 & 43,7 \\
\hline
\end{tabular}




\begin{tabular}{|l|c|c|c|c|c|c|c|}
\hline Par hab. en euros & 28,3 & 36,2 & 57,0 & 65,7 & 76,4 & 102,7 & 92,5 \\
\hline PNB par hab. & 1482 & 1674 & 1919 & 2101 & 2249 & 2498 & 2722 \\
\hline
\end{tabular}

Source : Banque nationale bulgare (BNB) et Institut national statistique (NSI) cités dans Vesselin Mintchev and Venelin Boshnakov, «Bulgarian Return Migration and Remittances : Alternative Estimates of Worker Remittances Inflow After 2000 », Working Paper Series, Sofia, 11 mai 2006.

Dans quelle mesure ces ressources contribuent-elles à modifier les modes de vie, pratiques de consommation et horizons d'attente tant des travailleurs que de leurs proches? Les sommes thésaurisées semblent avoir été jusqu'à présent majoritairement destinées à l'amélioration du quotidien, à l'achat de biens de consommation durables, à la réfection ou à la construction de logements ${ }^{31}$, plus rarement à l'investissement dans des activités entrepreneuriales ${ }^{32}$. De plus, l'aide apportée aux familles restées en Bulgarie tendrait à décroître au fur et à mesure que les migrants consolident leur situation légale, professionnelle et personnelle (avec la venue des conjoints et des enfants, voire avec de nouvelles naissances) dans leur nouveau lieu de résidence: l'étude réalisée par Eugenia Markova et Barry Reilly auprès de 198 Bulgares à Madrid, en novembre-décembre 2003 et en avril 2004, a ainsi suggéré l'existence d'une étroite corrélation statistique entre le statut juridique des migrants (légal ou illégal) et les montants envoyés en Bulgarie (plus conséquents tant que les personnes en mobilité vivent dans une situation précaire) ${ }^{33}$.

\section{4 - Les effets de l'adhésion de la Bulgarie à l'Union européenne}

Les traités d'adhésion de 2003 avaient offert aux Etats membres de l'UE la possibilité de restreindre l'accès des nouveaux entrants à leur marché du travail pendant une période maximale de sept ans (selon un principe $2+3+2$ ). Cette clause a été reprise dans les traités d'adhésion signés par la Bulgarie et la Roumanie deux ans plus tard. En 2004, seuls trois pays avaient décidé de n'imposer aucune restriction : la Grande-Bretagne, l'Irlande et la Suède. Au terme des deux premières années, en mai 2006, l'Espagne, la Grèce, la Finlande et la Portugal ont supprimé les barrières à l'entrée ; six autres pays ont décidé d'ouvrir aux nouveaux membres plusieurs secteurs d'activité confrontés à des déficits de main d'oeuvre (Belgique, Danemark, France, Italie, Luxembourg et PaysBas). L'Allemagne et l'Autriche ont en revanche maintenu leurs restrictions ${ }^{34}$. Un mois plus tard, l'Italie suivait l'exemple espagnol. En 2007, l'Allemagne et l'Autriche ont également facilité les embauches dans les spécialités confrontées à une pénurie de travailleurs.

On dispose désormais d'un recul suffisant pour apprécier les mouvements intervenus depuis l'Europe post-communiste après le $1^{\text {er }}$ mai $2004^{35}$ : fin 2006 , dans les 15 anciens Etats membres (E15), la part des actifs originaires des Etats centre-européens ayant rejoint l'UE (les E8) était inférieure à $1 \%$ de la main d'œuvre (elle s'établissait à $0,8 \%$ en Grande-Bretagne et $0,6 \%$ en Allemagne). Les pourcentages les plus élevés concernaient l'Irlande (2,4\%), l'Autriche (1,3\%) et le Luxembourg (1\%). Au total, 682500 citoyens des E8 étaient employés dans l'un des quinze anciens membres de l'UE, principalement en Grande-Bretagne (34\%, soit 233200 personnes) et en Allemagne (33,1\%, soit 226700$)^{36}$. La France n'a pas été une destination privilégiée, puisque seuls 15800 ressortissants des E8 y travaillaient à cette date. Toutefois, certains Etats - à l'image de la Grande- 
Bretagne et de l'Irlande - ont été confrontés à un afflux de migrants très supérieur aux anticipations, principalement des travailleurs polonais. Accentuée par le contraste entre leur politique migratoire libérale et celle des autres Etats membres européens, l'intensité de ces courants migratoires a conduit les deux pays à réviser leur position au moment de l'adhésion de la Bulgarie et de la Roumanie à l'UE ${ }^{37}$ : en octobre 2006, la Grande-Bretagne a décidé que les citoyens bulgares et roumains devraient disposer d'un permis de travail, s'inscrire sur les quotas de migrants temporaires dans l'agriculture et l'industrie agroalimentaire (respectivement 16250 et 3500 pour l'année 2007) ou quérir un emploi à travers les programmes spéciaux en faveur de la main d'oeuvre hautement qualifiée ${ }^{38}$. On notera cependant qu'en Grande-Bretagne comme en Irlande la mobilité post-2004 a été principalement de courte de durée : au printemps 2007, les premiers signes de retours vers les pays d'origine étaient d'ores et déjà enregistrés ${ }^{39}$. La crise économique de 2008 semblerait avoir accéléré ce mouvement en Grande-Bretagne, en Espagne et dans les autres Etats membres de l'UE.

En mai 2006, une enquête réalisée à la demande du ministère du Travail et des Affaires sociales bulgare estimait entre 45700 et 50000 le nombre des Bulgares envisageant de rechercher un emploi à l'étranger après le $1^{\text {er }}$ janvier $2007 ; 2 \%$ des personnes interrogées indiquaient souhaiter quitter le pays définitivement ${ }^{40}$. Bien qu'il soit encore tôt pour pleinement évaluer l'impact de l'accession de la Bulgarie à l'UE, les premières estimations disponibles révèlent des mouvements post-adhésion plus faibles que prévu : d'après les données fournies par Eurostat, en 2007 la Bulgarie aurait enregistré un solde migratoire négatif de 33000 personnes $^{41}$. Comme au cours des années précédentes, ces déplacements ont concerné prioritairement l'Espagne, l'Italie et la Grèce (en dépit du fait que ces Etats n'avaient pas opté pour une ouverture intégrale de leur marché du travail). Plusieurs facteurs contribuent à expliquer les recours limités à la mobilité. Le premier réside sans doute dans le fait que, pour la Bulgarie, le pic de migration a été atteint dans les années 2000-2004 - soit plusieurs années avant l'adhésion. Au moment de l'entrée dans l'Union, la majorité des citoyens bulgares aspirant à travailler à l'international étaient déjà en mouvement. Les transformations socio-économiques intervenues dans le pays constituent une seconde variable explicative : la Bulgarie a enregistré une croissance soutenue depuis 1999 couplée à un afflux d'investissements étrangers, puis à l'émergence de déficits ponctuels de main d'œuvre (dans les professions hautement qualifiées, d'une part, dans des secteurs d'activité très dynamiques comme la construction, d'autre part). Dans les grandes entreprises internationales implantées en Bulgarie, les salaires sont attractifs et le différentiel par rapport aux rémunérations d'Europe de l'Ouest va décroissant ${ }^{42}$. Les contrastes en matière de salaires demeurent en revanche sensibles dans les métiers moins qualifiés (transport, services, bâtiment, agriculture, tourisme, etc.).

21 La conjoncture économique représente un troisième paramètre. L'éclatement de la «bulle immobilière » en Espagne et la crise qui sévit depuis plusieurs mois en Europe ont déjà provoqué une diminution des mobilités, singulièrement dans l'agriculture et la construction. Il n'est pas à exclure en la circonstance que les ressortissants bulgares suivent la trajectoire de migrants roumains dont certains ont utilisé les savoir-faire acquis à l'étranger pour créer une entreprise en Roumanie ${ }^{43}$. Ce, d'autant plus que la Bulgarie est parvenue à maintenir un rythme de croissance soutenu dans la seconde moitié de l'année $2008^{44}$ et a - momentanément - joui d'un avantage comparatif par rapport à ses partenaires européens ${ }^{45}$. Une enquête réalisée en septembre 2008 à l'initiative du Ministère de Travail et des Affaires sociales bulgare auprès des migrants 
en Espagne suggérait toutefois que, même en cas de chômage (l'Espagne comptait 2,5 millions de chômeurs en août 2008, dont 500000 travailleurs étrangers ${ }^{46}$ ), la majorité d'entre eux n'envisageraient pas de revenir en Bulgarie. Deux raisons étaient avancées par les participants à l'enquête : la faiblesse des salaires bulgares dans leur secteur d'activité (35\% d'entre eux occupaient des emplois à faible qualification en Espagne) et les risques d'extension de la crise économique à la Bulgarie ${ }^{47}$. Le processus d'intégration à l'Union européenne n'a pas seulement influencé - fût-ce à un niveau moindre que ce que l'on attendait - les mobilités internationales des Bulgares. Il a également participé du développement de flux migrants à destination de la Bulgarie (ou transitant par elle) et a contribué à redéfinir son insertion dans son environnement régional. En adhérant à l'UE, la Bulgarie a dû reprendre à son compte l'intégralité de la "liste noire des visas » dressée par l'Union ${ }^{48}$. Dès 1999, elle avait introduit un système de visa pour les anciennes Républiques soviétiques sauf la Russie et l'Ukraine qui ont bénéficié d'une période de grâce jusqu'en octobre 2001. En revanche, les autorités bulgares s'étaient abstenues d'imposer des visas à la Macédoine et à la Serbie-et-Monténégro voisines ${ }^{49}$. Cette décision reflétait l'existence d'enjeux à la fois symboliques (la majorité des Bulgares entretient des sentiments de proximité à l'égard des Macédoniens, un peuple perçu comme ayant des origines communes avec les Bulgares) et économiques (les bénéfices retirés du commerce frontalier et du tourisme). Avant l'introduction des visas, entre 700000 et 800000 Macédoniens se rendaient chaque année en Bulgarie - un chiffre impressionnant rapporté à la population du pays (2 millions d'habitants) (voir tableau 2). Commerce de proximité, investissements mixtes, tourisme et attractivité des universités bulgares aux yeux des étudiants macédoniens expliquaient ces mouvements. Dans le cas de la Serbie-etMonténégro, les liens étaient moins soutenus mais leur renforcement progressif traduisait la découverte par les Serbes d'une destination touristique d'autant plus appréciée que le nombre des pays où ils pouvaient se rendre sans visa s'était effondré depuis la fin de la Yougoslavie. Dans un contexte d'isolement relatif, l'ouverture de la frontière bulgare avait acquis une valeur nouvelle.

Tableau 2 - Nombre d'entrées en Bulgarie de visiteurs de Macédoine et de Serbie-et-Monténégro entre 2000 et 2006

\begin{tabular}{|l|l|l|l|l|l|l|l|}
\hline Pays d'origine & 2000 & 2001 & 2002 & 2003 & 2004 & 2005 & 2006 \\
\hline République de Macédoine & 880000 & 859000 & 849000 & 887000 & 858000 & 755000 & 713000 \\
\hline Serbie et Monténégro & 512000 & 591000 & 841000 & 887000 & 853000 & 775000 & 827000 \\
\hline
\end{tabular}

Source : Institut national statistique (NSI), cité dans Angelina Tchorbadjiyska, «Bulgarian Experiences with Visa Policy in the Accession Process... », op. cit., p.96.

En amont de l'accession européenne, la Bulgarie a décidé la gratuité des visas et cherché à faciliter leur délivrance en ouvrant deux nouveaux consulats à Bitola (sud de la Macédoine) et à Niš (est de la Serbie). Mais ces mesures n'ont pas réussi à empêcher l'effondrement des contacts bilatéraux : au cours des premiers mois de 2007, les entrées depuis la Macédoine ne représentaient plus que 29,96\% de celles relevées l'année précédente $(28,75 \% \text { dans le cas de la Serbie })^{50}$. Dans les régions frontalières, le surcoût en termes de temps et d'énergie a eu raison de stratégies de survie qui avaient pourtant 
contribué à atténuer l'impact de la crise économique affectant les régions orientales de la Macédoine ${ }^{51}$. Le nombre des permis de résidence de longue durée accordés à des Macédoniens avait cependant, lui, augmenté (4 100 au cours des dix premiers mois de 2007 contre moins de 2800 en 2006) ${ }^{52}$.

Notons enfin que, bien que la Bulgarie reste un pays accueillant peu de travailleurs étrangers, de petites communautés migrantes - originaires de Chine, du Proche-Orient, ainsi que d'Afrique - se sont ces dernières années établies sur son territoire. Certaines y occupent des niches professionnelles spécifiques (tels les Chinois dans la restauration et le commerce en milieu urbain $)^{53}$. En 1993, l'Institut statistique national bulgare relevait la présence de 32000 citoyens étrangers ; en 1998, ils étaient $93000^{54}$. Au cours de la même période, 11000 étrangers ont reçu la citoyenneté bulgare. Ces données suggèrent le développement (encore modeste) d'une immigration en Bulgarie qui devrait se poursuivre dans les années à venir.

Une fois brossées à grands traits les principales mobilités bulgares depuis 1989, il convient maintenant de revenir plus en détail sur les conditions d'émergence des études migratoires en Bulgarie, ainsi que sur les interrogations autour desquelles ce nouveau champ s'est structuré.

\section{II - Etudier les migrations en Bulgarie : les constructions plurielles d'un objet de recherche}

En Bulgarie même, le développement des études sur les migrations intervient principalement au tournant du XXIème siècle ${ }^{55}$. Il mobilise des enseignants-chercheurs, des personnels d'organisations non gouvernementales (ONG), ainsi que des consultants travaillant pour des agences gouvernementales et/ou des commanditaires internationaux ${ }^{56}$. La désignation des migrations comme un "problème social $»^{57}$ imposant une "mobilisation coordonnée » des pouvoirs publics - dominante sur les scènes internationale et européenne - n'est pas étrangère au rôle des organisations internationales et des organismes publics dans le financement de ces travaux, tout comme à l'influence des approches décisionnelles au sein du nouveau champ. La distribution des acteurs ayant investi la thématique migratoire en Bulgarie reflète en même temps les profondes transformations intervenues, dans ce pays comme ailleurs en Europe, dans les définitions des métiers de la recherche, leurs modes d'organisation et leur condition de légitimation sociale. L'émergence d'une logique de « projets » dans lesquels les vertus managériales sont parfois plus importantes que la maitrise de savoirs méthodologiques et/ou empiriques, d'une part, la porosité croissante des rôles (chercheurs, consultants, prescripteurs de normes, décideurs...), d'autre part, ont peutêtre revêtu un caractère plus brutal en Bulgarie (en raison de la fermeture d'instituts de recherche publics, de la réduction drastique des aides étatiques, etc.) que dans des pays comme la France, mais ils n'en relèvent pas moins de processus partagés.

La richesse de travaux produits ces sept dernières années n'en est que plus remarquable ${ }^{58}$. Trois acteurs institutionnels ont joué un rôle-pivot en la matière : l'Académie des sciences bulgare (BAN, notamment sa Section d'ethnologie balkanique, l'Institut de sociologie, l'Institut d'études balkaniques, l'Institut d'histoire et l'Institut de démographie); l'université de Sofia "Kliment Ohridski» (Départements d'ethnologie, de sociologie, d'histoire et de théorie de la culture en particulier) et la 
Nouvelle université bulgare (Départements d'études politiques et d'anthropologie). Pardelà ces institutions, il convient de saluer les recherches menées et/ou financées par l'Institut pour l'étude des minorités et des relations interculturelles (Meždunaroden centăr za izsledvane na malcinstvata $i$ kulturni vzaimodejstvija, IMR) que dirige l'historienne ottomaniste, Antonina Željazkova. On doit notamment à IMIR d'importantes enquêtes qualitatives sur les migrations, mais aussi un travail majeur de diffusion et de valorisation de la recherche à travers la publication de thèses de doctorat. Le Center for Advanced Studies (CAS) de Sofia, une structure privée actuellement dirigée par l'historienne, Diana Miškova, joue par ailleurs depuis sa fondation un rôle remarquable de pépinière de la recherche ${ }^{59}$. Enfin, en dehors de Bulgarie, une nouvelle génération de chercheurs bulgares - souvent eux-mêmes forts d'une expérience migrante - est en train d'apparaître ${ }^{60}$.

Les modes de construction de l'objet «migrations " retenus reflètent les ancrages disciplinaires des chercheurs et, plus encore, les redéploiements intellectuels aux termes desquels les questions de mobilité ont été érigées en thèmes d'investigation. Certains universitaires - principalement des démographes et des économistes, plus rarement des sociologues - ont abordé les nouveaux courants migratoires dans le cadre de réflexions sur les transformations des tissus démographique, économique et social bulgares postérieures à la chute du communisme. D'autres analystes - au premier chef des ethnologues, anthropologues et historiens - sont venus aux enjeux de mobilités à partir d'un intérêt pour des minorités turque, bulgare musulmane et rom qui ont figuré parmi les grands acteurs des circulations de l'après-1989. Enfin la redéfinition des rapports entre la «Bulgarie», les «Balkans» et l'«Occident» a été au coeur d'interrogations - chez des politistes, sociologues et historiens - sur les territoires et la citoyenneté en un monde globalisé au sein desquels les migrations de travail, formes de mobilité parmi d'autres, ont été prises pour analyseurs.

Dès la première moitié des années 1990, plusieurs démographes et économistes envisagent les mobilités inaugurées à la faveur du changement de régime dans une perspective macrosociale, informée par les résultats du recensement de 1992. En 1994, la revue de démographie, Naselenie (Population) publie ainsi un dossier sur les migrations qui replace les mouvements de l'après-1989 dans une perspective historique plus longue ${ }^{61}$. En ce début de décennie, le phénomène migratoire est - on l'a vu principalement approché sous l'angle du brain drain. La perspective de l' «exode des cerveaux" sera abandonnée dans la seconde moitié des années 1990, laissant apparaître des configurations variées en fonction des professions et temporalités envisagées ${ }^{62}$. Des études fines ciblent alors des segments spécifiques de migrants potentiels, à l'instar de celle qu'Ivan Calăkov, de l'Institut de sociologie (BAN, section "Sociologie des sciences et éducation »), consacre entre 2000 et 2004 aux « Etudiants bulgares dans les technologies de l'information: attitudes envers l'émigration ou le travail en Bulgarie " grâce à un financement du ministère de l'Enseignement et de la Recherche ${ }^{63}$. Ces travaux sont contemporains de la réforme du système d'enseignement bulgare et des efforts de revalorisation, sur le marché du travail local, des diplômes acquis à l'étranger.

30 Au même moment, un nombre croissant d'experts internationaux investit la thématique du « risque migratoire » en provenance des pays ex-communistes dans le cadre de vastes enquêtes empiriques ${ }^{64}$. En Bulgarie même, les matériaux statistiques s'enrichissent (notamment après la réalisation du recensement 2001) tandis que se 
multiplient les sondages sur les «attitudes » migratoires des citoyens. Commandités par des acteurs tels que l'Organisation internationale pour les migrations (OIM), ces travaux s'emploient à dessiner les profils des migrants, leurs motivations, leurs itinéraires, les transferts monétaires qu'ils font parvenir à leurs proches, ainsi que les conditions d'un éventuel retour ${ }^{65}$. Sources statistiques et enquêtes d'opinion s'y côtoient au détriment de la collecte de matériaux qualitatifs (entretiens auprès des migrants, de leurs familles, de leur entourage, de leurs employeurs, observation participante, etc.). Les représentations sociales de l'ailleurs, les relations complexes entretenues avec les parents, amis et réseaux sociaux dans les lieux d'origine comme de résidence, les redéploiements des espaces et temporalités auxquels les va-et-vient donnent lieu restent en dehors de la focale ${ }^{66}$. Au début des années 2000, au sein des organisations internationales une attention émerge néanmoins pour une forme particulière de mouvements - les migrations pendulaires jugées caractéristiques de la période contemporaine. Dans l'étude qu'elles réalisent pour OIM en 2003, l'anthropologue, Rossitza Guentcheva, et la politologue, Petya Kabakchieva, s'attachent à approfondir la compréhension de ces circulations migratoires ${ }^{67}$.

31 Leur étude entre en écho avec une seconde gamme de travaux dont on pourrait dire qu'ils relèvent plus spécifiquement des "anthropologies du voyage " (Fariba Adelkhah) ${ }^{68}$ - appliquées ici aux migrations dites «ethniques ». Dans un pays dont la diversité de peuplement a été construite comme un enjeu par les élites politiques sur la moyenne durée, les questions minoritaires ont constitué un champ de recherche privilégié. Après 1989, l'exploration des proclamations identitaires et des relations intercommunautaires connait par ailleurs un nouvel élan en raison de l'héritage de l'assimilation forcée (1984-1989) ${ }^{69}$. Des ethnologues, historiens, anthropologues et, plus rarement, politistes s'attèlent à déchiffrer les conditions de rétablissement d'une coexistence intercommunautaire sereine. A l'Académie des sciences bulgare, dans des ONG comme IMIR, le Comité Helsinki bulgare (Bălgarski Helsinki komitet, BHK), ACCESS, SEGA, cette large communauté de chercheurs ${ }^{70}$ apporte à l'étude des itinéraires migrants sa sensibilité au terrain, ses méthodes qualitatives, son goût pour l'analyse microsociale des arts de faire et des représentations socioculturelles ${ }^{71}$.

Les trajectoires de mobilité de la communauté turque (qui a connu un exode à fortes résonances politiques et sociales en 1989) sont les premières à recevoir une attention suivie $^{72}$. Ces mouvements ne sont pas sans précédents historiques : depuis la création d'un Etat en Bulgarie et la sortie de facto de l'Empire ottoman en 1878, l'histoire des populations turques de Bulgarie a été marquée par une succession de vagues migratoires, tantôt désirées, parfois consenties, en d'autres circonstances forcées (1950-1951 et 1989 ${ }^{73}$. Les analyses des circulations du temps présent s'opèrent donc parallèlement à une relecture du passé et de la contribution des politiques publiques bulgares et turques aux mobilités des Turcs bulgares ${ }^{74}$. L'exploration des mouvements des Bulgares musulmans intervient quelques années plus tard. L'ethnologue Margarita Karamihova fait ici figure de précurseur, elle qui lance en février 2002 à l'Institut d'ethnologie de BAN une étude sur «Les attitudes migratoires de la population des Rhodopes » financée par IMIR ${ }^{75}$. Ce projet permet la réalisation d'un film documentaire et la publication d'un ouvrage collectif devenu depuis lors un classique où sont posés les premiers jalons d'une réflexion associant l'examen des circulations à une réflexion sur les redéfinitions des hiérarchies, appartenances et rôles induites par le fait migratoire ${ }^{76}$. Toujours à l'Institut d'ethnologie de BAN, mais cette fois-ci autour des ethnologues, Elena Marušiakova et Veselin Popov, se développent en parallèle des 
travaux sur les migrations des communautés roms ${ }^{77}$. Cette configuration institutionnelle et disciplinaire spécifique explique qu'aujourd'hui encore les mobilités des minorités (turque, musulmane et/ou rom) soient les mieux connues en Bulgarie ${ }^{78}$.

Les chercheurs spécialisés dans l'étude des minorités n'ont toutefois pas l'exclusive des interrogations sur les migrations et la renégociation des identités sociales et culturelles. Quelques sociologues et politistes ont abordé l'enjeu des mobilités depuis un questionnement sur les mutations des ordres stato-nationaux à l'heure de la globalisation et de l'intégration européenne. Les mises en mouvement des travailleurs bulgares y ont été envisagées comme l'une des mobilités d'un monde contemporain post-moderne (avec les séjours touristiques, les pèlerinages, les échanges universitaires, les missions d'experts, etc.). Dans leurs travaux, elles valent avant tout comme un lieu où saisir l'émergence de sensibilités et manières de penser inédites, corrélées aux processus de globalisation. La littérature post-moderne (Zygmunt Bauman, Arjun Appadurai) comme celle sur le transnationalisme fournissent ici des références intellectuelles très sollicitées ${ }^{79}$. L'entrée d'auteurs tels que Petya Kabakchieva et Ivaylo Ditchev dans le champ des migrations gagne aussi à être mise en relation avec l'importance qu'a revêtu, dans les sciences sociales bulgares des années 1990, le débat sur la "balkanéité » des Balkans et les relations symboliques Balkans/ Occident. L'ouverture de la Bulgarie post-communiste sur un espace régional en recompositions violentes, les interprétations orientalisantes des guerres d'exYougoslavie ont suscité de vifs débats en Bulgarie. L'ouvrage de l'historienne d'origine bulgare, Maria Todorova, Imagining the Balkans (1997) y a rencontré un écho d'autant plus ample que les grilles de lecture utilisées pour interpréter les destinées nationales bulgares n'étaient pas toutes, loin s'en faut, exemptes de déterminisme et de culturalisme $^{80}$. La préparation de l'adhésion à l'UE a par ailleurs relancé les interrogations sur une européanisation entendue comme modernisation. Dans leurs déplacements spatiaux - qui sont aussi des franchissements de démarcations culturelles et temporelles -, les migrants ont dès lors été vus comme acteurs ou, à défaut, comme sujets de ces « rencontres interculturelles».

A partir d'interrogations inscrites dans des champs de connaissance différents, trois débats se sont constitués ces dernières années. Ils concernent les vécus du départ et de l'éloignement, les contributions de la mobilité à un refaçonnage identitaire et les logiques de territorialisation/déterritorialisation. Abordant les migrations en tant qu'expériences humaines, certains textes mettent en exergue les souffrances attachées à des mobilités jugées synonymes de déclassement social et d'humiliations; la consommation ostentatoire pratiquée lors des brefs retours en Bulgarie, mécanisme compensatoire, serait impuissante à atténuer ces expériences ${ }^{81}$. D'autres écrits suggèrent l'existence de mécanismes complexes d'ajustement aux va-et-vient à la faveur desquels sont remodelés tant les lieux de résidence que les terroirs d'origine. L'accent est ici placé sur le caractère éminemment dynamique des identifications ${ }^{82}$. Plusieurs travaux soulignent également combien les mobilités ont été peu à peu incorporées à des définitions individuelles et collectives de l'existence "normale». Mila Mančeva évoque à cet égard la situation de migrants turcs bulgares installés à Berlin pour qui la dissémination des membres de leur famille entre la Bulgarie, la Turquie, l'Allemagne, les Pays-Bas, etc. tient lieu de fait social banalisé. Elle y voit « un processus de transnationalisation des familles migrantes des Turcs bulgares, qui préservent leur potentiel non seulement comme une ressource et une forme de soutien 
matériel et social, mais aussi comme structures de hiérarchies d'âge et de genre au sein desquelles sont prises les décisions importantes engageant la vie des membres ${ }^{83}$.

Cette remarque ouvre une deuxième discussion relative, elle, aux reformulations identitaires suscitées par les mobilités. Sur la base d'entretiens effectués auprès de migrants bulgares en Italie et en Bulgarie, Petya Kabakchieva déplore l'absence chez ces citoyens mobiles d'une "identité nationale clairement exprimée ${ }^{84}$. Elle en veut pour preuve le fait que les migrants déclarent ne rien regretter de la Bulgarie à l'exception de paysages, d'aliments, de musiques, ainsi que d'amis et de proches. Le jugement peut surprendre si l'on se souvient que les pratiques culturelles participent fondamentalement de la création d'identifications valorisantes à un "nous", de la matérialisation d'un être ensemble et que c'est précisément pour cette raison qu'elles se prêtent volontiers à une construction en emblèmes identitaires. Le vêtement, les arts de la table, les odeurs, certaines sonorités forgent des sensibilités qui sont partie intégrante des appartenances, et notamment de l'appartenance nationale. Autrement dit, le «national » ne se réduit pas à la revendication de droits collectifs, pas davantage à l'exercice de la citoyenneté, ni d'ailleurs aux lieux et moments de politisation des identités. Dans le sillage des travaux de Rogers Brubaker, il n'est pas sans intérêt de s'arrêter sur les modalités - réflexives ou non - d'identitarisation et de désidentitarisation (ces " intermittences " identitaires) des vécus quotidiens dont l'alimentation, le vêtement, la musique sont constitutifs ${ }^{85}$. Ces manières d'être et de se sentir appartenir, situationnelles et relationnelles, sont inscrites dans des expériences sociales dont l'intelligibilité est indispensable à une appréhension des constructions de soi. Enfin, l'on pourrait souhaiter rappeler que les valeurs auxquelles l'on se réfère pour fonder nos actions ne sauraient être, sans inventaire, tenues pour les moteurs de ces dernières ${ }^{86}$.

Plus largement, faut-il considérer que les expériences migratoires accentuent les identifications antérieures et renforcent les frontières entre "groupes ", qu'elles s'accompagnent d'un processus d'hybridation des appartenances ou encore qu'elles sont gérées à travers des basculements (switching) entre répertoires identitaires en fonction des situations? Pour Petya Kabakchieva, les mobilités mettent en jeu non la préservation d'une idée du "chez soi » et donc d'un territoire d'appartenance, mais plutôt la mise en flottement des identités et des lieux avec des mécanismes d'adaptation qui reposent sur l'alternance entre les codes d'« ici » et de «là-bas ». La perspective est fructueuse dans la mesure où elle rappelle le caractère contextuel et interactif des positionnements identitaires. Elle semble plus heuristique qu'une lecture qui tendrait à postuler la rigidité d'appartenances consolidées dans l'exposition à un Autre (oublieuse des différences dans les expériences migratoires, des trajectoires sociales contrastées des acteurs, des effets des apprentissages de la mobilité, etc.). Cependant, on pourrait lui préférer un regard plus attentif à la temporalité des parcours migrants et aux redéploiements identitaires qui s'y déroulent. Plus que d'un basculement entre codes - avec le postulat de stabilité que ces derniers impliquent - , il nous semblerait utile d'interroger des formes d'agrégation et de redéfinition de repères identitaires pluriels, de saisir la manière dont ils tiennent ensemble des individus dont les manières d'être peuvent différer dans le pays d'accueil et la terre d'origine, mais ne sauraient être réduits à une modalité unique dans l'un ou l'autre de ces espaces, ni être postulées pérennes. 

des mobilités, internes comme internationales, temporaires comme durables. Faut-il considérer que les acteurs mobiles formeraient, dans leur va-et-vient entre deux ou plusieurs Etats, des communautés post-nationales ou transnationales habitant des espaces relationnels à cheval sur plusieurs territoires ? Ou doit-on au contraire les envisager comme des individus flottants entre des ailleurs dont aucun ne serait réellement vécu comme sien ? La question de la spatialité est au cœur des travaux sur les faits migratoires. Pourtant, qu'il nous soit permis ici de suggérer une piste de recherche dont il nous semble qu'elle mériterait d'être explorée plus systématiquement - à savoir l'articulation entre le rapport au territoire et au temps et, plus généralement, la prise en compte dans les recherches sur les refaçonnages identitaires induits par les migrations de l'existence, en parallèle, de transformations contemporaines dans les appréhensions du temps. Depuis une vingtaine d'années, les historiens - dans le sillage d'un Reinhardt Koselleck, par exemple - ont souligné l'irréductible pluralisation des expériences du temps social ${ }^{87}$. Ils ont également décrit l'existence de changements majeurs dans les « régimes d'historicité ». François Hartog a relevé le développement d'un présentisme en rupture profonde par rapport aux imaginaires des articulations entre passé, présent et futur qui, depuis le XVIIIème siècle, ont structuré notre compréhension des mondes vécus et notre capacité à penser les discontinuités ${ }^{88}$. S'il est vrai que le tracé par des élites intellectuelles et politiques d'une ligne historique soustendue par le "Progrès » a représenté depuis deux siècles une condition de possibilité des ruptures tissées dans la redécouverte d'un passé mis en contemporéanité (ou encore approchées depuis l'avenir comme une projection du futur), quelles peuvent être les conséquences d'une remise en question de ces légitimations du mouvement? S'il est vrai que le tracé par des élites intellectuelles et politiques d'une ligne historique sous-tendue par le "Progrès " a représenté depuis deux siècles une condition de possibilité des ruptures tissées dans la redécouverte d'un passé mis en contemporéanité (ou encore approchées depuis l'avenir comme une projection du futur), quelles peuvent être les conséquences d'une remise en question de ces légitimations du mouvement?

Ces remarques suggèrent deux directions de recherche : d'une part, il ne serait pas sans intérêt d'interroger la manière dont les déplacements dans l'espace sont aussi, en même temps, vécus comme des déplacements dans le temps qui permettent parfois de ressaisir une idée de soi et d'envisager la projection vers l'avenir des existences individuelles et de groupe. D'autre part, mais l'invitation est ici plus intuitive et mériterait d'être travaillée, il pourrait être fructueux de chercher à rendre compte des incidences, sur les identifications, de la fin d'un moment historique (grossièrement du XVIIIème siècle à la fin du XXème siècle) ayant reposé sur la capacité à opérer une disjonction entre le passé et le présent au service d'une construction du futur. Si ces mécanismes d'appréhension du changement social ne sont plus opérants, s'il est vrai que nous sommes entrées dans une ère dominée par le présentisme, alors nos approches des modalités d'appartenance et de création d'un «nous » vont devoir être reconsidérées. Ces dernières années, maints travaux de sciences sociales ont attiré l'attention sur l'émergence d'acteurs affichant des identités de plus en plus nombreuses ; par-delà leurs épaisseurs historiques variées et leur degré d'institutionnalisation plus ou moins achevé, ces identités apparaissent fluides, infiniment « zappées » (et d'autant plus rigidifiées dans les discours des acteurs qu'elles peuvent être plus rapidement troquées contre d'autres). Les migrations - dans leurs mouvements multiples qui ne se réduisent d'ailleurs pas à un avant et un après, un « ici » et un " là-bas », mais, plus 
souvent, à une démultiplication des « ici » et des « là-bas » - pourraient fournir un des sites où observer les possibilités de (re)conceptualisation d'une unité d'expérience lorsque des acteurs vivent différemment, mais en même temps, des processus sociaux seulement partiellement similaires. A défaut, elles pourraient donner à voir les modalités de production de nouveaux ordonnancements identitaires aux ancrages spatiaux et temporels inédits.

Sans statuer sur ces enjeux, les articles rassemblés dans ce dossier devraient nous permettre d'affiner notre compréhension tant des ressources migratoires que des modalités d'articulation des vécus mobiles dans des identités culturelles et sociales retravaillées.

\section{III - Aborder les migrations depuis le microsocial : l'élaboration d'un dossier} principales motivations qui nous ont amenés, avec Elena Marušiakova et Veselin Popov, et en partenariat avec la Section balkanique de l'Institut d'ethnologie et du Musée ethnographique de l'Académie des sciences bulgare, à consacrer un dossier aux mobilités bulgares contemporaines en Europe. L'un de nos objectifs était de mettre en valeur l'apport de doctorants ou de jeunes docteurs ayant réalisé un travail de terrain souvent remarquable aux communautés scientifiques en Bulgarie. Les contributions présentées ici ont en commun de privilégier une démarche qualitative, attentive aux manières de faire, de ressentir et de dire le social. Les approches retenues sont pluridisciplinaires, tout en reflétant une sensibilité pour l'ethnologie, l'anthropologie et l'histoire.

Les articles sont ordonnés autour de trois polarités auxquelles ils ne se réduisent pas. Une première partie invite à inscrire les mobilités du présent dans un horizon temporel plus large parcouru de maillages spatiaux différenciés. Les excursions dans le temps suivent plusieurs voies: dans quelle mesure les mises en mouvement d'aujourd'hui s'inscrivent-elles en rupture ou en continuité - effective ou réinventée - par rapport aux mobilités communistes ou ante communistes? Les réactualisations du passé fournissent-elles des répertoires de sens permettant de penser les choix migratoires contemporains ou doivent-elles être plus prosaïquement envisagées comme des viviers de savoir-faire et de réseaux sociaux? Jusqu'à quel point, d'ailleurs, l'exploration de nouveaux espaces participe-t-elle d'un apprivoisement de l'aujourd'hui, d'un effort pour interpréter des mutations politiques, économiques et sociales dépassant le seul fait migratoire? Creuser les temporalités du mouvement invite ainsi à saisir, d'une part, la pluralisation des pratiques sociales du temps, d'autre part, les manières dont les transformations de l'après-1989 viennent plier et feuilleter des passés dont la proximité avec le présent est redéfinie.

Petko Hristov nous emmène ainsi sur les routes du gurbet ${ }^{89}$ - ces circulations de travail caractéristiques des Balkans ottomans à la faveur desquelles des migrants balkaniques monnayaient leurs savoir-faire d'agriculteurs et de bâtisseurs -. Source de revenus précieuse, le gurbet a également constitué un vecteur de remodelage des moments-clés de la vie sociale, ainsi que des expériences du territoire. Contrairement aux migrations contemporaines, le gurbet que nous décrit Petko Hristov est gendered (confiné pour

Balkanologie, Vol. XI, n 1-2 | 2008 
l'essentiel aux hommes) ; il s'inscrit dans la durée d'une saison économique et n'engage pas la perspective d'une installation définitive. L'une des richesses de l'article réside dans la description fine du voyage comme passage (à l'âge adulte) et de la manière dont les présents gèrent l'absence en la symbolisant. Les départs, les retours se guettent à travers les traces qui les rendent possibles et dans lesquelles ils sont dits: les calendriers festifs familial et villageois, les cycles de vie (les mariages notamment) sont réorganisés pour épouser les rythmes du gurbet, tandis que les frontières entre l'ici et l'ailleurs sont marquées à travers la tristesse et les pleurs de la séparation à la lisière des villages. Nous voici d'entrée plongés au cœur des interactions entre individuation et appartenance au groupe qui jalonneront l'ensemble du dossier.

Pour Petko Hristov, la sortie de l'Empire ottoman s'est accompagnée moins d'une rupture que d'une réorientation du gurbet. Il voit dans les migrations de Gastarbeiter à destination de l'Allemagne et des pays d'Europe du nord des années 1970, comme dans les mouvements de l'après-1989, une déclinaison nouvelle de logiques pérennes. La comparaison entre migrations du début du XXème siècle et dynamiques postcommunistes présente l'avantage de nous rappeler que des phénomènes parfois trop hâtivement qualifiés de nouveaux (sans que les termes de cette nouveauté ou les lignes de démarcation entre l'avant et le maintenant soient explicités et discutés) mériteraient d'être mis en regard avec celles observées dans d'autres configurations historiques - non pour postuler l'existence de similitudes, mais pour mieux saisir d'éventuelles singularités (parmi celles-ci figurent sans doute des ordres territoriaux marqués par la généralisation du modèle de l'Etat-nation et des politiques publiques de contrôle des frontières et des statuts de citoyenneté/d'étranger renforcé, etc.) ${ }^{90}$. Dans une telle démarche, il pourrait être utile d'établir une distinction entre les modes d'énonciation discursive des mobilités (des termes identiques peuvent désigner des pratiques sociales innovantes), leurs conditions d'intelligibilité par ceux qui y prennent part (la référence à des imaginaires sociaux anciens et à une longue durée historique peuvent servir de repère afin d'appréhender le présent, $\mathrm{y}$ compris dans ses changements, voire pour mettre en œuvre ces métamorphoses) et les éventuelles continuités observées dans les formes et ressorts des mobilités contemporaines.

Dans leur contribution, Elena Marušiakova et Vesselin Popov, qui désignent également les migrations contemporaines sous le nom de gurbet, ne choisissent pas entre ces interprétations. Mais ils invitent à repenser les découpages temporels du XXème siècle traditionnellement proposés dans l'historiographie des migrations. Leur article se concentre sur un groupe spécifique, lui-même hétérogène - les Roms originaires de Bulgarie, de Roumanie et de Yougoslavie. Afin d'éclairer les circulations d'aujourd'hui, ils proposent un détour par une histoire rom traversée, depuis leur arrivée en Europe au XVème siècle, de "vagues migratoires " successives. De leur point de vue, la principale rupture en la matière ne date pas de 1989 et de la chute du communisme, mais bien des années 1960-1970 qui voient s'amorcer une troisième migration historique, singulièrement depuis la Yougoslavie. Adopter cette chronologie n'est pas sans implication pour l'analyse : la compréhension des mobilités n'est plus recherchée dans le seul changement des régimes politique et économique à l'Est ; elle est proposée à l'intersection entre la transformation des politiques migratoires de pays ouesteuropéens, elle-même imputable aux premiers frémissements d'une globalisation des marchés du travail, et les stratégies économiques et démographiques adoptées par les Etats communistes. Pays capitalistes et non capitalistes se retrouvent ici englobés dans 
une histoire commune qui dépasse des lignes de démarcation entre blocs «Est » et "Ouest ».

On ne peut toutefois s'empêcher de songer que la configuration qu'ils décrivent n'éclaire pas au même degré l'ensemble des trajectoires des Etats socialistes : quelle distance, en effet, entre l'encouragement yougoslave aux migrations du travail et la politique d'autarcie adoptée par l'Albanie ou encore la fermeture de la Roumanie des années 1980. L'article n'en apparaît pas moins précieux en ce qu'il nous permet de relier des périodes (ante communiste, communiste, post-communiste) dont toutes ne nous sont pas connues au même degré. Ainsi que Ralitza Soultanova ${ }^{91}$ et Rossitza Guentcheva (dans ce dossier) l'ont justement souligné, les décennies communistes font trop souvent figure d'angle mort dans les recherches sur les migrations, alors même que voyages professionnels, formations universitaires, séjours touristiques, échanges interétatiques de main d'œuvre ont fourni aux citoyens bulgares des expériences de l'ailleurs ayant affecté tant leurs vécus du socialisme que la réalisation des projets migratoires de l'après 1989.

La seconde partie du dossier nous propose l'exploration d'itinéraires migratoires singuliers, ceux des Turcs bulgares en Turquie (Mila Maeva); des Roms bulgares en Espagne (Magdalena Slavkova) et des citoyens bulgares en Grèce (Nikolaj Gabărski, Aliki Angelidou). Par-delà leurs différences, ces contributions éclairent d'abord les modalités pratiques du transit - deux thématiques dont Stéphane de Tapia a rappelé qu'elles restent encore à la périphérie des études sur les migrations $-{ }^{92}$, les réseaux sociaux qui facilitent la mobilité et ceux qui se constituent à la faveur des déplacements, les modes d'insertion sur les marchés du travail locaux, ainsi que le rôle des politiques migratoires des Etats receveurs dans les choix d'installation - temporaire ou permanente - des migrants. Ces textes nous fournissent ensuite une première série d'indices pour apprécier les contributions du de l'éloignement et des retours à la reformulation des appartenances individuelles et collectives, à la fabrique des statuts sociaux, ainsi qu'à la constitution d'espaces relationnels entre lieux d'origine et de résidence.

Mila Maeva nous propose d'accompagner les Turcs de Bulgarie dans leurs trajets vers un pays, la Turquie, pensé comme une "mère patrie ». Elle décrit les difficultés du voyage, mais surtout la rencontre entre des migrants minoritaires en Bulgarie - qui espéraient s'insérer dans l'espace territorial et symbolique plus vaste de la nation turque - et des citoyens de Turquie pour qui ces nouveaux venus sont aussi, voire avant tout, des travailleurs migrants. Les modes d'entrée sur le marché du travail (dans des conditions initialement précaires), de logement (en périphérie des grandes villes) et de vie au quotidien (fondée sur la thésaurisation) contribuent à faire de cette rencontre un lieu producteur de "différences ». Turcs en Bulgarie, ils sont parfois considérés comme «Bulgares» (bulgarlar) en Turquie, au point de s'y ressentir une fois de plus comme une minorité. Les interactions font saillir les contrastes dans les rapports à l'islam (les Turcs de Bulgarie jugent les Turcs de Turquie plus «conservateurs »), le rôle de la femme, mais aussi les pratiques festives et alimentaires. Elles s'opèrent en parallèle avec un réancrage symbolique dans une région d'origine autour de laquelle s'ordonnent les sociabilités reconstituées en terre de migration. Le lien densifié n'est pas celui qui unit à la Bulgarie en tant qu'Etat ni même à une idée de la bulgarité citoyenne, mais celui qui scelle le rapport physique, sensoriel et symbolique avec un «chez soi » situé en Bulgarie. 

migrants magyars de Transylvanie partis travailler en Hongrie après $1989^{93}$ et amenés à renégocier les définitions d'eux-mêmes et de la "mère-patrie » à l'intersection entre des assignations extérieures - liées non seulement à des univers culturels différents mais aussi à des positions socio-économiques spécifiques au sein de la société hongroise - et des identifications internes marquées par l'expérience de la distance sociale avec les citoyens de Hongrie ${ }^{94}$. Par rapport à la trajectoire des Magyars de Transylvanie, plusieurs éléments de spécificité peuvent être cependant notés : pour la majorité des migrants turcs de Bulgarie, le déplacement depuis leur terre natale vers la Turquie s'accompagne aussi d'un exode rural. Issus d'un univers essentiellement rural, ils s'installent en effet dans des centres urbains pourvoyeurs d'emplois. Le sentiment d'étrangeté, la nostalgie de la terre quittée sont aussi ceux de paysages de montagnes, de collines ou de terres agricoles remplacés par des cités bruyantes de vie. Les politiques des kin states (Hongrie et Turquie) envers leurs minorités de l'extérieur contribuent également à la singularisation de chaque configuration : Mila Maeva souligne que tous les informateurs qu'elle a rencontrés détenaient la double nationalité (bulgare et turque), alors que nombreux sont les migrants hongrois en Hongrie dépourvus de passeport hongrois. La majorité des migrants turcs de Bulgarie dont l'auteur a suivi les trajectoires se sont par ailleurs installés de façon durable, si ce n'est définitive en Turquie, quoique cette installation n'engage pas une disparition totale des liens avec la région de naissance. Seule une frange des migrants a pris le parti de revenir en Bulgarie. Enfin, dans les imaginaires croisés des Turcs de Bulgarie et des citoyens de Turquie, une dernière variable contribue à introduire un écart par rapport à la situation des Hongrois de l'extérieur: le processus d'intégration à l'Union européenne (UE). Là où la Hongrie et la Roumanie sont devenues membres de l'UE, quoiqu'à quelques années de décalage, seule la Bulgarie a rejoint l'Union à ce jour. Grand pays, imaginé plus prospère que la Bulgarie, plus "avancé » aussi, la Turquie s'est trouvée repositionnée symboliquement plus à l'Est au fur et à mesure que son voisin balkanique se rapprochait d'un horizon "européen». La référence à «l'européanité » était déjà mobilisée par les migrants turcs bulgares dans leurs relations avec les citoyens de la "mère patrie", notamment pour valoriser des pratiques cultuelles et religieuses jugées plus «modernes ", l'adhésion européenne de la Bulgarie a contribué à renforcer les usages de ces échelles symboliques qui font du migrant, minoritaire et souvent socialement fragilisé, un acteur culturellement " supérieur ».

contribution de Magdalena Slavkova nous propose un double déplacement - vers un autre "groupe» et une autre destination - elle qui s'intéresse aux expériences migratoires des Roms de Bulgarie en Espagne. Elle souligne l'importance de se départir de deux assomptions erronées : la première réside dans l'idée selon laquelle l'insertion des Roms dans des réseaux migratoires traduirait la redécouverte du nomadisme par des populations sédentarisées de force pendant la période socialiste. Non seulement, dans le contexte bulgare, la part des Roms ayant mené une existence nomade au regard des populations sédentarisées était modeste mais, en outre, les recours à des stratégies de survie mobiles s'observent aussi bien parmi les sédentaires que dans les groupes ayant autrefois nomadisé. Le second postulat dont il convient de se dépendre est celui qui consisterait à supposer l'existence de solidarités immédiates, évidentes, fondées sur une commune appartenance "rom » entre les groupes désignés de l'extérieur comme tels. En migration, nous explique-t-elle, les distinctions entre les diverses communautés 
roms de Bulgarie sont maintenues (à travers les modalités de déplacement, les itinéraires et destinations choisies, voire le logement dans le pays de destination). De la même manière, il n'y a aucune solidarité automatique entre Gitanes d'Espagne (qui occupent une place généralement supérieure à celle des nouveaux venus sur l'échelle sociale), Roms de Roumanie (plus nombreux, plus visibles aussi dans l'espace public en raison de la préservation - chez certains d'entre eux - de codes vestimentaires distinctifs, qu'on ne retrouve pas à l'identique dans les groupes roms migrants venus de Bulgarie) et Roms de Bulgarie. Le tissage des liens n'est sensible que parmi les Roms convertis à l'évangélisme - pour ces derniers, l'engagement religieux fournit d'ailleurs une ressource clé dans les parcours de mobilité - et c'est alors principalement autour des pratiques religieuses communes et des réseaux sociaux qui y sont attachés que Gitanes, Roms roumains et Roms bulgares se retrouvent, non en raison d'une ethnicité partagée.

L'analyse proposée par Magdalena Slavkova jette également un éclairage précieux sur les appartenances valorisées en migration. Les définitions de l'individu et du groupe s'adossent à des démarcations dont certaines sont relativement pérennes (comme la délimitation entre Roms et non-Roms), mais nouent de manières complexes des interactions entre sous-groupe, "ethnicité » et nationalité. L'identification au pays d'origine et, dans une certaine mesure à une identité bulgare, nous dit-elle, sort doublement renforcée des mises en mouvement en raison du rôle que joue la nationalité dans le traitement des migrants par les autorités espagnoles, d'une part, et du regard porté sur les Roms de Roumanie au sein de la société espagnole, d'autre part. Cette présentation des strates identitaires plurielles - redéfinies les unes et les autres en interaction avec des acteurs publics et des interlocuteurs au quotidien - n'est pas sans affinité avec celle proposée par Mila Mančeva dans son étude sur les réseaux de solidarité « co-ethniques » (des Turcs d'Allemagne) mobilisés par les Turcs bulgares en Allemagne. Si son terrain l'amène à observer le rôle des solidarités communautaires dans la recherche d'un logement ou d'un emploi, par exemple, elle rappelle que ces dernières reflètent moins l'existence d'un sentiment d'appartenance "ethnique " commun (les différences culturelles entre les groupes étant également produites et/ou mises à jour dans des interactions conditionnées par leurs rôles sociaux et par la position singulière des Turcs allemands en Allemagne) qu'une structuration spécifique des communautés migrantes, de la politique migratoire et du marché du travail en Allemagne.

51 Sur les modes de présentation de soi, les rapports entretenus avec espaces de résidence et d'origine, les deux textes consacrés aux migrations bulgares en Grèce jettent un éclairage complémentaire. Une des premières destinations des migrations bulgares après 1989, la Grèce a vu sa position au sein des Balkans bouleversée par la fin de la guerre froide, tandis que des espaces frontaliers, longtemps interdits à la circulation, redevenaient lieu de passage et de solidarités adossées à la mobilisation de passés communs. L'analyse que nous propose Nikolaj Gabărski explore au premier chef les migrations bulgares en milieu rural, particulièrement dans la région de Pelasgia ; Aliki Angelidou, elle, a posé son regard sur la capitale, Athènes, et sa périphérie urbaine. Dans la première configuration, le travail agricole saisonnier occupe une place de choix dans les stratégies d'emploi adoptées par les migrants. Dans la configuration que nous décrit Aliki Angelidou, les services à domicile, la construction constituentles principaux débouchés des travailleurs migrants. Les deux auteurs se rejoingnent pour souligner les 
phénomènes de déclassement social corrélés à l'exercice de métiers faiblement qualifiés.

52 Avec Nikolaj Gabărski, nous sommes invités à suivre les migrants à travers chacune des étapes qui les conduisent depuis leur village ou ville d'origine jusqu'à leur destination en Grèce. Il retrace le développement des réseaux de passeurs (les kanaldžii) dont les services furent massivement sollicités avant la levée des visas Schengen, dessine les voies de passage entre les deux pays et déroule le récit de la recherche d'emploi par les migrants. Contrairement à ce que l'on aurait pu anticiper, ses recherches de terrain suggèrent par ailleurs que le rôle des passeurs n'a pas disparu au moment de la levée des visa Schengen en avril 2001 : les modalités des migrations n'ont changé que plus tardivement, à la fois en raison des conditions de mise en œuvre des nouvelles dispositions par les douaniers grecs et de l'attachement des migrants à des pratiques et interlocuteurs devenus familiers.

Dans la contribution d'Aliki Angelidou, nous retrouvons les migrants bulgares installés dans la précarité d'emplois peu qualifiés et, surtout, invités à dire la rencontre avec l'Autre, les sentiments - changeant dans le temps - de proximité et de distance, de familiarité et d'inconnu. Chacun des entretiens qu'elle a réalisés avec des travailleurs migrants nous ramène aux questionnements abordés plus haut: la circulation sur des terres européennes dont l'altérité culturelle, le degré «d'avancement » économique sont perçus comme impliquant un déplacement de soi dans le temps, aide-t-elle à apprivoiser le présent en lui offrant la perspective d'un devenir individuel tendu vers le progrès? Pour les migrants bulgares, la découverte de la Grèce - un pays qui était envisagé avant le voyage comme un "nous en plus avancé » - passe par plusieurs étapes dont chacune implique des repositionnements du soi au regard d'étalons de valeur changeants. Au fil du temps, cet Ailleurs de la Grèce prend les formes - successives ou coexistantes - d'un «nous » en mieux, d'un « Autre ", d'un " moins bien que nous ». Les confrontations plurielles à un écart culturel parce que social, modulé en fonction du statut des migrants (irrégulier ou non, précaire ou consolidé, avec ou sans maîtrise de la langue locale) s'accompagnent de relocalisations de soi sur une échelle temporelle (développé/retardé) qui n'en finit pas d'être également spatiale et culturelle. Dans le même temps, la circulation s'impose, à la lecture des entretiens d'Aliki Angelidou, également comme un moyen pour donner sens à des expériences de vie, y compris celles antérieures à la mise en mouvement. Se déplacer permet alors de trouver, de marquer sa "place »- laquelle ne se pense pas seulement dans les termes de la réussite sociale, de l'interconnaissance et de la reconnaissance, mais aussi comme une sensation en son corps.

Ce que suggère le texte d'Aliki Angelidou est dès lors qu'il nous faut, dans un troisième et dernier temps, revenir - géographiquement et symboliquement - vers la Bulgarie. Rossitza Guentcheva suit cette voie en nous proposant un premier " détour » (au sens de Georges Balandier) par les mobilités internes à la Bulgarie et les politiques publiques destinées à les encadrer. Sa contribution s'ouvre sur une interrogation fondatrice : de quel espace et de quelles circulations parle-t-on lorsque l'on travaille sur les migrations? Il est de coutume, dans les études des faits migratoires, de se concentrer sur les seules "migrations internationales", celles qui traversent les frontières étatiques. Ce sont ainsi paradoxalement les milieux de recherche les plus prompts à remettre en question dans leurs travaux la pertinence des frontières stato-nationales (à travers l'utilisation de notions comme le transnationalisme, par exemple) qui réifient 
ces mêmes frontières en considérant que migrations "internes» (vătrešni) et "externes» (vănsni) devraient de relever de traitements distincts. Et si les déplacements internationaux ne constituaient que la poursuite, l'expansion des logiques de circulation existant à l'intérieur des espaces stato-nationaux, comment faudrait-il penser les interrelations entre ces deux types de mouvements? A l'instar des travaux sur les migrations roumaines, Rossitza Guentcheva souligne les ressources que peuvent représenter de premières migrations internes en vue d'expériences migratoires ultérieures à l'étranger. Les apprentissages de la mobilité, l'incorportation des circulations dans des parcours de vie commencent souvent au sein d'un espace régional.

Sa contribution est par ailleurs riche d'une autre proposition de rupture méthodologique : en interrogeant aussi bien les pratiques migratoires que le mode de traitement, par la puissance publique bulgare, des migrations internes avant et après la chute du communisme, elle avance la nécessité de remettre en question le postulat d'une rupture en 1989 et, corrélativement, celui d'une alterité, d'une exceptionnalité socialistes. Dans les formes de mobilités elles-mêmes, l'auteur souligne l'existence de permanences sensibles : la tendance à la réduction de l'exode rural, par exemple, avait été amorcée dès les années 1960. La propension de l'Etat à vouloir encadrer, infléchir les flux migratoires apparait elle aussi inscrite dans la moyenne durée: ce sont principalement les moyens qui ont changé (plus d'interdictions, mais des incitations), non l'ambition de contrôler. Les objectifs portent désormais moins sur la gestion de la rareté des biens publics dans les centres urbains que sur l'encouragement libéral à une flexibilisation de la main d'œuvre. Ils s'inscrivent dans l'affichage d'un idéal étatique et européen soumis à de nouvelles rationalités. Attentative aux continuités à l'intérieur des discontinuités, l'auteur attire cependant l'attention sur l'émergence d'un phénomène qui, à défaut d'être statistiquement significatif, n'en apparaît pas moins qualitativement intéressant - à savoir le retour vers les villages (mais pas nécessairement vers un mode de vie agricole) de populations urbaines confrontées à une crise des ressources ne leur permettant plus d'assumer les dépenses liées à un mode de vie urbain.

Observant les nouvelles dynamiques migratoires internationales depuis la Bulgarie, Rossitza Guentcheva nous propose enfin un éclairage original de la manière dont se redéploient les liens entre l'ici et l'ailleurs. Son article dessine en effet les contours d'une dichotomie possible entre l'étranger, lieu de mobilités plurielles et de travail, et une Bulgarie parfois vouée à une immobilité consommatrice. Certains migrants qui ont connu un emploi à l'étranger (et les rémunérations "occidentales" afférentes) ne peuvent plus envisager de travailler localement pour le salaire très inférieur qui leur serait proposé. Ils élaborent alors des modes d'existence bipolaires dans lesquels les séjours dans la terre d'origine s'égrènent dans l'épuisement progressif de l'épargne accumulée grâce au détour par l'international avant de nouveaux départs au-delà des frontières. Mobilités transnationales et immobilités bulgares deviendraient ainsi les deux faces d'une même réalité sociale.

Avec l'article de Margarita Karamihova, la palette des indices, des impressions, glanées tout au long du dossier viennent se réordonner en un ensemble des processus individuels et sociaux, générationnels et de genre, identitaires et culturels, qui sont d'autant mieux saisis dans leurs imbrications que l'auteur a choisi de consacrer son étude à un site spécifique, le village de Satovča, dans les Rhodopes occidentaux (au Sud- 
Ouest de la Bulgarie), où habitent une majorité relative de Bulgares musulmans, des Bulgares orthodoxes et des Roms musulmans pour certains récemment convertis à l'évangélisme. Avec cette recherche d'une très grande richesse ethnographique, le prisme d'analyse ne porte plus sur les lieux de destination des migrants, ni sur les itinéraires migratoires des citoyens en mobilité ; il se concentre sur l'espace du village natal, celui précisément dont Margarita Karamihova nous montre à quel point il est parvenu, au prix de transformations et différenciations nouvelles, à s'imposer comme le «noyau modal » d'une territorialité imaginée élargie aux «filiales » d'Espagne, de Grèce ou des Etats-Unis. Ce déplacement de focale présente au moins deux avantages pour l'analyse : il nous aide d'abord à nous départir de la propension, fréquente dans les études sur les migrations, à imputer aux faits migratoires la majorité des mutations de rôle, d'identités et de statuts observées dans des lieux où de larges segments de la population sont entrés en mobilité. Margarita Karamihova décrit très justement les interactions entre plusieurs séries de changements liées à la transformation de l'économie de subsistance locale, au réordonnancement de l'ordre politique postcommuniste, au processus d'intégration européenne...et à des mises en mouvement multiples (migrants saisonniers, migrants de longue durée, jeunes partis suivre une formation en théologie islamique à l'étranger, etc.).

L'adoption d'un ancrage territorial spécifique nous rappelle ensuite les risques auxquels se heurte toute tentative de généralisation - à des groupes sociaux ou ethnoculturels particuliers ou à des espaces régionaux ou nationaux - d'observations sur les dynamiques migratoires. Elle permet également une plus grande sensibilité aux inflexions temporelles. Si le développement des circulations à Satovča dans la seconde moitié des années 1990 a ainsi contribué à la fabrication d'une "mythologie du succès migratoire " associant aux départs à l'étranger des images d'initiative, du force de caractère ou de réussite, ces représentations - qui ne furent jamais univoques - ont, nous rappelle Margarita Karamihova, connu une dévaluation rapide. Vers 2005, les migrations avaient cessé d'être pensées comme le vecteur premier de l'accomplissement de soi ou de la redéfinition des hiérarchies sociales. Tandis que les migrants de retour au village peinaient à traduire leur expérience de la mobilité spatiale en une mobilité sociale au sein de leur environnement d'origine - en dépit d'investissements dans des propriétés ou dans une consommation ostentatoire -, localement la stabilisation de la situation économique, un plus grand accès au crédit et surtout les bouleversements associés aux identifications musulmanes avaient déjà commencé à forger de nouveaux critères de définition des statuts.

Très attentif aux réseaux sociaux - notamment de lignages - et à la manière dont ils sont mobilisés et redéfinis au cours des expériences migratoires, le travail de Margarita Karamihova nous donne en effet à voir la manière dont les ressources symboliques, sociales et identitaires de l'islam peuvent faire partie intégrante des renégociations des hiérarchies internes au village. La formation reçue dans des pays arabes par de jeunes Bulgares musulmans parvenus à occuper des positions au sein des institutions religieuses et écoles locales, la mise en œuvre concurremment par l'Etat d'une politique encourageant l'enseignement des religions, ont contribué à placer les investissements dans le répertoire confessionnel au service d'un renversement des hiérarchies symboliques entre ceux qui migrent et ceux qui restent. Aux femmes demeurées au foyer pendant que les hommes partaient travailler au loin, l'adoption d'une nouvelle lecture de l'islam - qui conjugue les arguments de l'enracinement dans une tradition à ceux de la modernité et de la réussite sociale des puissances arabes du Proche-Orient - 
permet de négocier un rôle social et une autonomie décisionnelle renforcés. Plus largement, les habitants restés au village peuvent trouver dans le répertoire de la «vraie » foi le moyen de se projeter dans les espaces mobiles et immenses du monde musulman. A contrario, les migrants - dont les stratégies de survie à l'étranger peuvent passer par ce que Margarita Karamihova qualifie de «mimétisme identitaire ", à savoir le non-affichage dans l'espace public des pays d'accueil de l'identité musulmane peuvent se retrouver socialement disqualifiés par ce que certains des nouveaux prêcheurs musulmans, les "savants ", présentent comme un éloignement de la foi. La reconquête d'une position sociale prestigieuse pour ceux qui sont partis en migration peut dès lors passer soit par une intériorisation des nouvelles normes religieuses, soit par un évergétisme social dans la sphère confessionnelle (la contribution financière à la construction d'une nouvelle mosquée, par exemple).

$\mathrm{Au}$ terme de ces parcours foisonnants, volontiers insaissisables, à travers certaines des migrations bulgares en Europe, ce dossier aura rempli son rôle s'il est parvenu à susciter, au sein d'un public de lecteurs et de chercheurs francophones, le désir de prolonger les recherches sur la Bulgarie et sur ses citoyens mobiles. Avant de clore cette introduction, il reste seulement à remercier tous les participants au dossier pour leur soutien et leur enthousiasme, ainsi que Bernard Lory, Miladina Monova et Sandrine Bochew pour leur participation à la traduction des articles bulgares.

\section{NOTES}

1. Voir Diminescu (Dana) (dir.), Visibles mais peu nombreux. Les circulations migratoires roumaines, Paris : Ed. de la Maison des sciences de l'homme, 2003, p.1-24 (esp. p.1-2).

2. Voir, par exemple, Leyard (Richard), Blanchard (Olivier), Dornbush (Rudiger), Krugman (Paul), East-West Migration Alternatives, Cambridge : MIT Press, 1992. Une étude menée en 1998 par l'Organisation internationale pour les migrations (OIM) dans onze pays de l'Est s'étonnait de la faiblesse des aspirations au départ en Bulgarie. Voir IOM Technical Cooperation Centre for Europe and Central Asia, "Migration Potential in Central and Eastern Europe », Geneva : IOM, 1999, à l'adresse : http://iom.ramdisk.net/iom/images/uploads/ IOM\%20TCC\%20Rep_1071067863.pdf (p.65-69)

3. Iordan Kalčev (démographe), Daniela Bobeva, Veselin Minčev (économistes) et Ivan Čalăkov (sociologue) font exception à cet égard qui, dès le début des années 1990, ont interrogé les mouvements migratoires du point de vue de leur impact démographique et économique sur la Bulgarie.

4. Ce mode d'interprétation des migrations gagnerait à être mis en regard avec l'existence, en Bulgarie, d'imaginaires sociaux de la nation organisés autour d'une peur de l'effritement, voire de la disparition - que ce soit à travers la perte d'un segment de territoire, la " débulgarisation » des émigrants ou encore l'existence de différentiels démographiques entre «Bulgares ethniques » et représentants des minorités.

5. Sur ce phénomène social, voir Kaneff (Deema), « Holiday Location or Agricultural Village? British Property Owners in Rural Bulgaria », Eastern European Countryside, 12, 2006, p.79-92. 
6. Voir à ce sujet la recherche de Marina Liakova sur les étudiants bulgares dans la Ruhr. Cf. Liakova (Liakova), «Hibridnijat žiznen svjat na bălgarskite studenti v Germanija » [L'univers hybride des étudiants bulgares en Allemagne], Kritika i Humanizăm (Critique et humanisme), 25, 2008, p.45-63.

7. 503 jeunes avaient pris part à la première réunion de la Pâque bulgare à Sofia, évènement depuis lors routinisé. Ils étaient plus de mille cinq cents l'année suivante, en provenance de 23 pays. Voir Weekly Government Bulletin, 24-20 avril 2000, à l'adresse : http://212.122.160.99/old/ eng/gis/bulletin/

2000/24_28_April.htm\#503\%20Bulgarians\%20Registered\%20in\%20the\%20Initiative\%20\%93Bulgarian\%20Easter\%94\%20until\%20April\%2

8. Sur l'enjeu de « retour », voir Vasilev (Georgi), « Văzmožnija koridor za zavrăštane - Formi i varianti » [Un corridor possible pour le retour - formes et variantes], Sofia: Agence pour les Bulgares à l'étranger, 2002, à l'adresse : http://www.aba.government.bg/bg/pages/Izsledvaniya/ Reintegration/

2\%D0\%9C\%D0\%B8\%D0\%B3\%D1\%80\%D0\%B0\%D1\%86\%D0\%B8\%D1\%8F\%D1\%82\%D0\%B0

9. Pour reprendre les catégories utilisées par les acteurs publics bulgares. On remarquera que celles-ci décrivent assez mal un fait migratoire nourri des circulations multiples et de départs récurrents.

10. Les principales destinations privilégiées ont été l'Allemagne, la Suède et l'Autriche. Entre 1989 et 1993, environ 96000 citoyens bulgares ont déposé une demande d'asile dans des Etats ouest-européens, dont $78 \%$ en Allemagne. Voir UNHCR Centre for Documentation and Research, Background Paper on Bulgarian Refugees and Asylum Seekers, Geneva : UNHCR, 1994, cité dans August Gächter \& International Labour Office (ILO), "The Ambiguities of Emigration : Bulgaria since 1988 », International Migration Papers, 39, 2002, p.23-24, Geneva, à l'adresse : http:// www.ilo.org/public/english/protection/migrant/download/imp/imp39.pdf ; sur le cas spécifique de l'Allemagne, voir aussi Mančeva (Mila), « Trudova migracija na bălgarskite turci v Germanija. Sǎetnični migrantski mreži i kulturi » [La migration de travail des Turcs bulgares en Allemagne. Réseaux et cultures migrants co-ethniques], Kritika i humanizăm (Critique et humanisme), 25 (1), 2008, p.25-44 (p.2-4).

11. Voir Soultanova (Ralitza), «Les migrations multiples de la population bulgare », Actes du colloque «La France et les migrants des Balkans : un état des lieux», 20 janvier 2005, Paris, Courrier des Balkans, à l'adresse : http://balkans.courriers.info/article5504.html

12. Voir Kabachieva (Petya), "Temporary Migrants: Beyond Roles, Across Identities", in : Kiossev (Aleksander), Kabachieva (Petya), eds., Rules and Roles : Fluid Institutions, Hybrid Roles and Identities in East European Transformation Processes (1989 - 2005), Freiburg : LitVerlag (à paraître). Une tri-partition proche (settlers, migrants de moyen terme/plus d'un an, migrants de courte durée/moins d'une an) est retenue dans Mintchev (Vesselin), Boshnakov (Venelin), Kaltchev (Jordan), Goev (Valentin), "Who is leaving? Potential emigration from Bulgaria in the beginning of the XXI century», 2003, à l'adresse: http://www.cerge-ei.cz/pdf/gdn/ RRCIII_28_paper_01.pdf

13. 87895 en 1990, 46496 en 1991, 69348 en 1992, 66426 en 1993. Chiffres cités dans Bobeva (Daniela), Čalăkov (Ivan), Markov (Jordan), Migracijata - evropejskata integracija i iztičaneto na mozăci ot Bălgarija [La migration : l'intégration européenne et la fuite des cerveaux en Bulgarie], Sofia : Center for the Study of Democracy (CSD), 1996, p.19, à l'adresse : http://www.csd.bg/ artShowbg.php?id=6823

14. Voir Vassileva (Darina), « Bulgarian Turkish Emigration and Return », International Migration Review, 26(2), été 1992, p.342-352.

15. Hadjiisky (Magdaléna), Les migrations est-européennes dans le contexte de la transition : l'exemple de l'émigration bulgare, DEA d'études soviétiques et est-européennes, Paris : Sciences Po, 1993.

16. Les chercheurs en sciences sociales ont plutôt entrepris des reconversions vers l'administration; maints spécialistes en ingénierie et en techniques se sont tournés vers 
l'économie privée. Voir Bobeva (Daniela), Čalăkov (Ivan), Markov (Jordan), Migracijata evropejskata integracija i iztičaneto na mozăci ot Bălgarija, op. cit., p.15 et suiv.; Cekova (Evgenja), «Zagubite na naučen potencial ot zvenata na BAN prez 1990-1992 » [Les pertes de potentiel universitaire depuis BAN en 1990-1992], Naselenie (Population), 4, 1993, p.93-96.

17. Daniela Bobeva et al. ont reconstitué les trajectoires de 544 chercheurs. Près de la moitié d'entre eux avaient pu suivre une spécialisation à l'étranger avant 1989. La voie migratoire a été choisie par $22,8 \%$ des personnels diplômés d'un pays occidental (13,3\% de ceux formés dans un pays du bloc socialiste et $11,3 \%$ en Bulgarie). Ibid.

18. Sur le commerce de la valise en milieu rom, voir Konstantinov (Yulian), « Hunting for Gaps Through Boundaries : Gypsy Tactics for Economic Survival in the Context of the Second Phase of Post-totalitarian Changes in Bulgaria ", European Journal of Social Sciences, 7(3), 1994, p.237-248. Concernant les trajectoires turques, voir Parla (Ayşe), « Irregular Workers or Ethnic Kin?: Post 1990s Labor Migration from Bulgaria to Turkey ", International Migration 45(3), 2007, p.157-181.

19. Cf. le documentaire Les indésirables. La minorité turque en Bulgarie,réalisé par Adela Peeva, ARTE/ZDF, 2000, 55 minutes.

20. En contrepartie, l'Allemagne négocie avec la Bulgarie, comme avec d'autres Etats postcommunistes, des accords bilatéraux de réadmission. Voir Angenendt (Stephen), «L'asile et l'immigration en Allemagne », Politique étrangère, 59(3), automne 1994, p.731-748.

21. Guild (Elspeth), Bigo (Didier), « Schengen et la politique des visas », Cultures \& Conflits, 49, printemps 2003, à l'adresse : http://www.conflits.org/index921.html.

22. On note dans le même temps, à partir du milieu des années 1990, un développement des migrations en direction du continent américain (Etats-Unis et Canada), favorisé dans le cas des Etats-Unis par l'existence du système de «loterie » pour l'obtention des green cards. 5411 Bulgares ont pu en bénéficier en 1998, 4181 en 1999, 4381 en 2000, 2893 en 2001, 2489 en 2002 et 2843 en 2003, 3482 en 2004, 4068 en 2005, 2131 en 2006, 1674 en 2007 et 1154 en 2008. Chiffres officiels disponibles à l'adresse: http://greencardlottery.visapro.com/Green-CardLottery-Results.asp

23. Le passage de mobilités temporaires à une installation définitive peut cependant intervenir en cas d'obtention de permis de séjour et de travail, de réunion familiale et/ou de naissance d'un enfant. Voir Morokvasic-Muller (Mirjana), «La mobilité transnationale comme ressource : le cas des migrants d'Europe de l'Est », Cultures et conflits, 33-34, 1999, p.105-122.

24. Voir Rau (Victor), Mesini (Béatrice), «Segmentation statutaire et ethnique du marché de l'emploi en agriculture : le cas des saisonniers migrants dans la production de fruits et légumes en Méditerranée». Communication à la conférence «Nouvelles dynamiques migratoires: activités régulières et irrégulières sur le marché du travail européen », Université de Nice Sophia Antipolis, 6-8 décembre 2007, p.14, p.15, à l'adresse: http://www.unice.fr/migractivities/ 04_SousPression.pdf

25. Voir Sunderhaus (Sebastian), Regularization Programs for Undocumented Migrants, VDMVerlag, 2007.

26. Les élargissements européens de 2004 et de 2007 n'ont pas remis en cause l'«utilitarisme migratoire ». Sur la base d'une étude réalisée dans le secteur fruit et légumes de la région PACA, au sud de la France, Victor Rau et Béatricie Mesini soulignent que, pour la première fois en 2004, le nombre des migrants saisonniers polonais (6640) a quasiment égalé celui des Marocains (6 909). Voir Rau (Victor), Mesini (Béatrice), « Segmentation statutaire et ethnique du marché de l'emploi... ", op. cit., p.14.

27. Les recherches menées par Mila Mančeva ont suggéré que la levée des visas en avril 2001 avait réduit la durée moyenne des séjours des migrants turcs bulgares en Allemagne. Le ralentissement du secteur de la construction, la hausse des prix consécutive à l'adoption de l'euro et la répression accrue, à partir de 2004, du travail non déclaré ont par ailleurs réduit 
l'attractivité de l'Allemagne au profit des Pays-Bas, de l'Espagne et du Portugal. Voir Mančeva (Mila), « Trudova migracija na bǎlgarskite turci v Germanija... », op. cit., p.4-5.

28. Mintchev (Vesselin), Boshnakov (Venelin), « Bulgarian Return Migration and Remittances : Alternative Estimates of Worker Remittances Inflow After 2000 », Bulgarian Academy of Sciences - Institute of Economics \& University of National and World Economy (UNWE), Sofia, 11 mai 2006, p.7, à l'adresse : http://papers.ssrn.com/sol3/cf_dev/AbsByAuth.cfm?per_id=371392.

29. Op.cit., p.4.

30. Ibid., p.10 et 11 .

31. Une recherche menée par l'Institute for Market Economics (Institut za pazarna ikonomika, un think tank libéral) sur le marché de l'immobilier dans plusieurs grandes villes bulgares a ainsi suggéré que quelque $10 \%$ des biens acquis entre 2002 et 2004 avaient été financés grâce aux envois d'argent des migrants. Cité dans Markova (Eugenia), Reilly (Barry), « Bulgarian Migrant Remittances and Legal Status : Some Micro-level Evidence from Madrid », South-Eastern Europe Journal of Economics, 5(1), 2007, p.57.

32. Dans la littérature, les liens entre remises des travailleurs migrants et développement économique des pays d'origine ont fait l'objet d'études innombrables aux résultats ambivalents. A l'échelle des Balkans contemporains, plusieurs recherches empiriques consacrées aux Albanais ont suggéré que les revenus issus des séjours à l'étranger étaient consacrés à l'alimentation, au vêtement, à la restauration des biens et propriétés, qu'ils étaient faiblement thésaurisés et plus rarement encore réinvestis dans des activités productives. Les migrations circulaires auraient de plus suscité le développement d'habitudes de consommation inédites qui favorisent les importations (depuis l'Occident) au détriment de la production locale. Une fois le bénéfice du travail à l'étranger épuisé, les citoyens albanais repartiraient en migration. Cf. Arrehag (Lisa) et al., "Cross-border Migration and Remittances in a Post-Communist Society: Return Flows of Money and Goods in the Korçë District, Albania », South Eastern Europe Journal of Economics, 1, 2005, p.9-40; Castaldo (Adriana), Reilly (Barry), « Do Migrant Remittances Affect the Consumption Patterns of Albanian Households?», South Eastern Europe Journal of Economics, 1, 2007, p.25-54; Gedeshi (Ilir), «Role of Remittances from Albanian Emigrants and their Influence on the Country's Economy », Eastern European Economics, 40(5), 2002, p.49-72.

33. Markova (Eugenia), Reilly (Barry), «Bulgarian Migrant Remittances and Legal Status : Some Micro-level Evidence from Madrid ", South-Eastern Europe Journal of Economics, 5(1), 2007, p.55-71.

34. Voir European Commission, "Enlargement, Two Years After. An Economic Evaluation », Occasional Papers, 24, May 2006, p.80, http://ec.europa.eu/economy_finance/publications/ publication7548_en.pdf. Pour une présentation plus détaillée des débats au sein des E 15 et des mesures adoptées par chacun des Etats de l'UE, voir Doyle (Nicola) et al., Freedom of Movement for Workers from Central and Eastern Europe, Experiences in Ireland and Sweden, Stockholm: Swedish Institute for European Policy Studies (SIEPS), 5, May 2006, p.24-27, à l'adresse: http:// ec.europa.eu/enlargement/pdf/5th_enlargement/facts_figures/20065_en.pdf

35. Voir, notamment, European Action Citizen Service (ECAS), Who's Afraid of the EU's Latest Enlargement? The Impact of Bulgaria and Romania joining the Union on Free Movement of Persons, ECAS, 2008; E. Hönekopp, Labour migration studies in nine countries. General background and first attempt to present findings and proposals, Rapport présenté à Budapest à la conférence «European Cooperation in Labour Migration : Search for Best Practices", le 12 juin 2007 ; Doyle (Nicola), Hughes (Gerard), Wadensjö (Eskil), «Freedom of Movement for Workers from Central and Eastern Europe. Experiences in Ireland and Sweden », Report 5, mai 2006, Swedish Institute for European Policy Stuides (SIEPS); Barrel (Ray), Fitzgerald (John), Riley (Rebecca), «EU Enlargement and Migration : Assessing the Macroeconomic Impact », NIESR Discussion Paper, 292, mars 2007, Londres : National Institute of Economic and Social Research (NIESR).

36. Chiffres cités dans European Action Citizen Service (ECAS), Who's Afraid of the EU's Latest Enlargement? op. cit., p.4. 
37. Voir Institute for Public Policy Research (IPPR), Bulgaria and Romania - Migrations Implication for the UK, EU Enlargement FactFile, avril 2006, à l'adresse : www.ippr.org/members/ download.asp?f=/ecomm/files/eu_enlargement_factfile.pdf\&a=skip

38. Les étudiants et les personnes travaillant à leur compte ont été dispensés de ces obligations. Voir House of Commons. Trade and Industry Committee, Europe moves East : The Impact of the new EU Member states on UK business. Eleventh Report of Session 2006-2007, London: House of Commons, 18 october 2007, p.8.

39. Une enquête du Home Office, menée sur la période 2004-2006 à la faveur de l'inscription des nouveaux employés dans le cadre du Worker Registration Scheme, a ainsi suggéré que plus de 55\% des ressortissants des E8 inscrits n'entendaient pas rester en Grande-Bretagne plus de trois mois. L'Institute of Public Policy Research (IPPR), un think tank britannique, a par ailleurs montré que, dès la fin 2006, près de 140000 citoyens originaires des nouveaux membres avaient déjà quitté la Grande-Bretagne, soit approximativement $42 \%$ des migrants arrivés en 2004 . Une conclusion similaire peut être trouvée dans: Office of the Committee for European Integration, Department of Analyses and Strategies, Four years Poland's Membership in the EU, Analysis of Social and Economic Benefits and Costs, Varsovie, 2008, à l'adresse: http://ec.europa.eu/ enlargement/pdf/5th_enlargement/facts_figures/4_years_poland_en.pdf

40. Voir les résultats de l'étude sur les «attitudes migratoires" préparée par BBSS Gallup International pour le ministère du Travail et des Affaires sociales en septembre 2006, citée dans ECAS, op. cit., p.7

41. Voir Eurostat, "Données en bref, Premières estimations démographiques pour 2007 ", Population et conditions sociales, 3/2008, p.1, à l'adresse : http://epp.eurostat.ec.europa.eu/cache/ ITY_OFFPUB/KS-QA-08-003/FR/KS-QA-08-003-FR.PDF

42. Quelques branches restent plus exposées à un exode des cadres (les secteurs médical et dentaire, par exemple).

43. Voir Bran (Mirel), "L'Occident n'est plus le paradis qui nous faisait rêver ", Le monde, 22 octobre 2008.

44. La croissance bulgare a été de $6,7 \%$ au deuxième trimestre 2008. Chiffres de la Banque nationale bulgare (BNB), à l'adresse: http://www.bnb.bg/bnb/home.nsf/fsWebIndex? OpenFrameset

45. Les prévisions de croissance du gouvernement bulgare pour l'année 2009 ont été revues à la baisse et seront sans doute inférieures à $2 \%$.

46. Cité dans « Ispanija spira naemaneto na rabotnici ot Bălgarija » [L'Espagne arrête l'embauche de travailleurs de Bulgarie], Dnevnik, 4 septembre 2008.

47. Gutsuzjan (Mišel), «Emigrantite stavat trudovi nomadi » [Les migrants deviennent des nomades du travail], Dnevnik, 29 septembre 2008. A cette date, le nombre des Bulgares travaillant en Espagne était officiellement estimé à 120000 personnes (officieusement, à environ 200000 ).

48. Voir Council Regulation (EC) No 539/2001 of 15 March 2001 listing the third countries whose nationals must be in possession of visas when crossing the external borders and those whose nationals are exempt from that requirement, à l'adresse :http://eur-lex.europa.eu/smartapi/cgi/ sga_doc?smartapi!celexapi!prod!CELEXnumdoc\&lg=EN\&numdoc=32001R0539\&model=guichett.

49. Cf. Tchorbadjiyska (Angelina), «Bulgarian Experiences with Visa Policy in the Accession Process : A Story of Visa Lists, Citizenships and Limitations on Citizens' Rights ", Regio, 2007, à l'adresse : http://www.ceeol.com/aspx/getdocument.aspx?logid=5\&id=dee85631-bf49-40d6-a3eba8acbec3a53f

50. Chiffres de l'Institut national statistique, cités dans Tchorbadjiyska (Angelina), « Bulgarian Experiences with Visa Policy in the Accession Process.., op. cit., p.99.

51. Le petit commerce entre les deux pays, la vente sur les marchés permettaient à des Macédoniens de gagner quelque 130 euros par mois, dans un Etat où le salaire moyen est d'environ 250-300 euros, mais où le taux de chômage avoisine les 35\%. Voir Karajkov (Risto), 
«Europe's Visa Policy for the Balkans », World Press, 5 février 2007, à l'adresse: http:// www.worldpress.org/Europe/2662.cfm

52. Chiffres cités dans SOPEMI, International Migration Outlook 2008, Paris: OECD, 2008, à l'adresse: http://www.oecd.org/dataoecd/55/33/41252991.pdf. Les Turcs et les Britanniques occupent respectivement les seconde et troisième places pour ce qui concerne le nombre de permis de résidence de longue durée délivrés en 2007.

53. Krăsteva (Anna), "Kitajci» [Chinois], in Krăsteva (Anna), (dir.), Imigracijata v Bălgarija [L'immigration en Bulgarie], Sofia : IMIR, 2005, p.105-136.

54. Chiffres cités dans August Gächter \& International Labour Office (ILO), « The Ambiguities of Emigration: Bulgaria since 1988 ", International Migration Papers, 39, 2002, Geneva, p.9, à l'adresse : http://www.ilo.org/public/english/protection/migrant/download/imp/imp39.pdf

55. L'après-1989 est aussi marqué par la redécouverte des «Bulgares de l'extérieur » vivant en Ukraine, Moldavie, Russie, Slovaquie, etc. Dès les premières années des changements, des ethnologues et sociologues ont entrepris une exploration de leurs modes de vie, coutumes et identifications. Le changement de régime a en outre suscité la publication d'ouvrages consacrés aux émigrations politiques consécutives à l'avènement du communisme.

56. Ces postures ne sont pas mutuellement exclusives. Les situations de multi-positionnalités sont ici fréquentes.

57. Au sens où l'entend Edelman (Murray), Constructing the Political Spectacle, Chicago: University of Chicago Press, 1988.

58. La conférence organisée en juin dernier à Sofia offre un bon panorama des recherches en cours. Voir Migration in and from Southeastern Europe. International conference With the support of the Federal Ministry of Science and Research of the Republic of Austria in the framework of its SEE science cooperation initiative, Sofia, 2-3 juin 2008. Voir aussi le dossier consacré par la revue Kritika $i$ Humanizăm (Critique et humanisme) aux migrations $(25,2008)$.

59. C'est notamment dans le cadre du projet de recherche de CAS consacré aux « Roles, Identities and Hybrids» (2003-2006) que Petya Kabakchieva a développé sa réflexion sur les migrations. Voir Kabakchieva (Petya), «Crossing Borders : Changing Roles, Changing Identities (Temporary Migration as a Form of Socio-Cultural Exchange in the Enlarged EU)», Research Paper, Sofia : Center for Advanced Studies (CAS), 2006, à l'adresse: http://v3.cas.bg/cyeds/downloads/ CAS_RIH_Kabakchieva.pdf

60. Sans prétendre à l'exhaustivité, on mentionnera les travaux d'Eugenia Markova (London School of Economics, Londres), de Dimitrina Mihaylova (Université d'Oxford), de Mariana Ljakova (Université d'Essen) et d'Elena Jileva (Center for Political and Constitutional Studies, Madrid). Parmi les doctorants ou jeunes docteurs, se distinguent les recherches de Neda Deneva (Central European University, Budapest), de Ralitza Soultanova (Université libre de Bruxelles), d'Albena Tcholakova (Université de Lyon II) et de Magdalina Dzhamdzhieva (Université de Neuchâtel). Cet univers de recherche est, notons-le, très gendered...

61. Voir Naselenie (Population), 1, 1994.

62. L'approche des migrations en terme de «fuite des cerveaux " n'a pas été absente non plus des préoccupations de la Commission européenne ou encore de l'Organisation internationale du travail. Cf. European Commission, Migration - Europe's Integration and the Labour Force Brain Drain from Central and Eastern Europe, Brussels : European Commission, COST, 1997; Beleva (Iskra), Kotzeva (Mariana), Skilled Labour Migration from Developing Countries: Study on Bulgaria, Geneva : International Labour Office (ILO), 2001.

63. Pour plus de détails, voir http://www.sociology-bg.org/display.php? page=project\&cat=1\&article=7; Galev (Todor), Čalăkov (Ivan), "Naglasi za emigracija sred studentite ot informacionni i komunikacionni tehnologii v Bălgarija » [Attitudes en faveur de l'émigration parmi les étudiants bulgares en information et en technologies de la communication], Nauka (Science), 6, 2006, p.21-27. 
64. Voir, entre autres, Bauer (Thomas), Zimmermann (Klaus), "Assessment of Possible Migration Pressure and its Labour Market Impact Following EU Enlargement to Central and Eastern Europe ", IZA Research Report, 3, 19999; Tito Boeri and Herbert Bruecker, « Eastern Enlargement and EU Labour-Markets : Perceptions, Challenges and Opportunities », IZA DP, 256, 2001; Stephen Drinkwater, «Go West? Assessing the Willingness to Move from Central and Eastern European Countries », University of Surrey (UK), Department of Economics, à l'adresse: www.econ.survey.ac.uk/sdrinkwater/go-west.pdf

65. Kalchev (Jordan), Profile and motives of potential migrants from Bulgaria, Budapest : IOM, 1997; International Organization for Migrations, Migration Potential in Central and Eastern Europe, Geneva : IOM, 1999; International Organization for Migration (IOM)-Sofia, Migration Potential in Bulgaria, Sofia : IOM, 2001.

66. Cf. le bilan dressé dans August Gächter \& International Labour Office (ILO), "The Ambiguities of Emigration : Bulgaria since 1988 ", International Migration Papers, 39, 2002, Geneva, à l'adresse: http://www.ilo.org/public/english/protection/migrant/download/imp/imp39.pdf. A l'époque, les travaux de Eugenia Markova, concernant les migrants bulgares en Grèce, figurent parmi les toutes premières recherches qualitatives. Voir Markova (Eugenia), Sarris (Alexander), "The Performance of Bulgarian Illegal Migrants in the Greek Labour Market ", South European Society \& Politics, 2 (2), 1997, p.57-77.

67. Guentcheva (Rossitza), Kabakchieva (Petya), Kolarski (Plamen), «Bulgaria: The Social Impact of Seasonal Migration ", in: Sharing Experience: Migration Trends in Selected Applicant Countries and Lessons Learned from the 'New Countries of Immigration' in the EU and Austria, Vol I. Vienna : International Organization for Migration (IOM), 2003, à l'adresse : http://www.iom.int/ DOCUMENTS/PUBLICATION/EN/IOM_I_BG.pdf

68. Voir la journée d'étude "Migrations et anthropologie du voyage » organisée par Fariba Adelkhah et Jean-François Bayart au CERI, le 5 octobre 2005 et également Adelkhah (Fariba), Bayart (Jean-François), eds., Voyages du développement : émigration, commerce, exil, Paris : Karthala \& CERI, 2007.

69. Cf., au sein d'une très riche littérature, les deux volumes édités par l'ONG ACCESS sous le titre Aspekti na etnokulturnata situacija $v$ Bălgarija [Aspects de la situation ethnoculturelle en Bulgarie], Sofia : AKSES, 1992 et 1993 ; Zhelyazkova (Antonina ), éd., Relations of Compatibility and Incompatibility between Christians and Muslims in Bulgaria, Sofia: IMIR, 1995. Dans ce dernier ouvrage, Aleksandăr Antonov envisage les migrations (intra et interrégionales) depuis les régions de Zlatograd et de Assenovgrad. Des études ultérieures souligneront la contribution de ces premières expériences de mobilité à l'acquisition des savoir-faire ultérieurement utilisés dans le franchissement de frontières internationales. Cf. Antonov (Alexander), «Migration and Migration Process in the Regions of Zlatograd and Assenovgrad», in : Zhelyazkova (Antonina), éd., Relations of Compatibility and Incompatibility between Christians and Muslims in Bulgaria, Sofia: IMIR, 1995, p.323-331.

70. On peut songer aux travaux d'Antonina Zhelyaskova, directrice d'IMIR, mais aussi - par ordre alphabétique et sans prétendre à l'exhaustivité - à ceux de Mirella Dečeva, Valeri Grigorov, Petko Hristov, Margarita Karamihova, Mila Maeva, Mila Mančeva, Elena Marušiakova, Domna Mihajl, Veselin Popov, Valeri Rusanov, Valeri Stojanov, Ilona Tomova, Evgenija Troeva, Anastasija Vodeničarova.

71. Un même glissement depuis l'ethnicité vers les migrations peut être relevé dans le cas des recherches sur l'immigration. Le titre donné par Anna Krăsteva (qui dirige le CERMES à la Nouvelle université bulgare et a été un des principaux promoteurs de cette thématique) en 2004 au premier ouvrage qu'elle a consacré aux immigrants (Ot etničnost kăm migracija - De l'ethnicité aux migrations) est à cet égard éloquent.

72. Voir l'excellent Zhelyazkova (Antonina), éd., Between Adaptation and Nostalgia: The Bulgarian Turks in Turkey. Sofia: International Center for Minority Research and Intercultural Relations 
(IMIR), 1998. L'ethnologue Mila Maeva a consacré sa thèse de doctorat aux migrations turques bulgares en Turquie, une thèse publiée par IMIR. Voir Maeva (Mila), Bălgarskite turci-preselnici v Republika Turcija (kultura i identičnost) [Les Turcs bulgares installés en République de Turquie (culture et identité)], Sofia : IMIR, 2006.

73. Voir Stojanov (Valeri), Turskoto naselenie v Bălgarija meždu poljusite na etničeskata politka [La population turque en Bulgarie entre les pôles de la politique ethnique], Sofia : Lik, 1998.

74. En Turquie également, des travaux remarquables ont été réalisés sur les migrations des Turcs de Bulgarie. Songeons, par exemple, à Parla (Ayşe), «Irregular Workers or Ethnic Kin? Post nineties labour migration from Bulgaria to Turkey ", International Migration 45(3), août 2007, p.157-181; Parla (Ayşe), « Longing, Belonging and Locations of Homeland among Turkish Immigrants from Bulgaria ", Journal of Southeast European and Black Sea Studies 6(4), 2006; Parla (Ayşe), « Marking Time along the Bulgarian-Turkish Border ", Ethnography 4(4), 2003, p.561-575. 75. Un an plus tôt, Margarita Karamihova avait amorcé un autre terrain de recherche à l'occasion d'un voyage touristique aux Etats-Unis (du rôle des mobilités des chercheurs dans les études de la mobilité...). Cette enquête, poursuivie de 2001 à 2003, a fait l'objet d'une publication dans Karamihova (Margarita), Amerikanski mečti.Pătevoditel sred părva generacija imigranti [Les rêves américains. Guide chez les immigrants de première génération], Sofia : IK 'Krotal', 2004.

76. Karamihova (Margarita), éd., Da živeeš tam, da se sănuvaš tuk. Emigracionni procesi v načaloto na XXI vek [Vivre là-bas, se rêver ici. Processus migratoires au début du XXIème siècle], Sofia : IMIR, 2003.

77. Marushiakova (Elena), Popov (Vesselin), Decheva (Mirella), « The Gypsies in Bulgaria and their Migrations ", in Guy (Will), Uherek (Zdenek), Weinerova (Renata), eds., Roma Migration in Europe : Case Studies, Münster : Lit, 2004, p.135-142. Marušiakova (Elena), Popov (Veselin), « De l'Est a l'Ouest. Chronologie et typologie des migrations tsiganes en Europe ", Etudes tsiganes, 27-28, 2006, p.10-26 ; Marušiakova (Elena), Popov (Veselin), « La mobilité des Tsiganes dans la fédération des Etats indépendants ", Etudes tsiganes, 27-28, 2006, p.28-43 ; Slavkova (Magdalena), "Săvremenni trudovi migracii na bălgarski cigani kăm Ispanija i tiahnoto otraženie vărhu identičnostta im » [Les migrations de travail contemporaines des Roms bulgares à destination de l'Espagne et leurs répercussions sur leur identité], in Dečeva (Mirella), éd., Dinamika na nacionalnata identičnost $i$ transnacionalnite identičnosti $v$ procesa na evropeiska integracija [Dynamique de l'identité nationale et des identités transnationales dans le processus d'intégration européenne], Sofia : Paradigma, 2008.

78. En parallèle, a été redécouverte la thématique du gurbet, particulièrement investie par des chercheurs comme Petko Hristov. Ce regain d'intérêt a d'abord pris la forme d'un « Atelier sur le gurbet ", initié par Margarita Karamihova à l'automne 2001 à l'Institut d'ethnographie. Récemment, un projet de recherche bulgaro-macédonien associant la Section d'études balkaniques de l'Institut d'ethnographie de BAN en Bulgarie à l'Institut pour l'histoire nationale et l'Institut du folklore en Macédoine a été lancé autour du thème des « Dynamiques de la mobilité du travail dans les Balkans » (2008-2009).

79. On notera que l'influence $d u$ «transnationalisme» et des cultural studies postmodernes excède les cercles des politistes et sociologues. Elle est sensible dans la majorité des travaux sur les migrations, toutes disciplines confondues.

80. Voir les écrits d'Aleksandăr Kiossev sur ce qu'il a appelé les « cultures auto-colonialisées ». Kiossev (Alexander), « Notes on the Self-Colonizing Cultures », in Pejic (B.), Elliott (D.), eds., Art and Culture in post-Communist Europe, Stockholm: Moderna Museet, 1999, p.114-118; Kiossev (Alexander), The Dark Initimacy : maps, identities and acts of identification, à l'adresse: http:// kolektiviza.wordpress.com/test/the-dark-intimacy-maps-identities-acts-of-identificationalexander-kiossev/.

81. Cf., par exemple, Ditchev (Ivaylo), «Le citoyen mobile et l'ombre de son identité », in Rautenberg (Michel), éd., La Bulgarie et l'Europe, Paris : L'Harmattan, 2007, p.165-178. 
82. Voir, entre autres, les contributions de Margarita Karamihova, Mila Maeva, Magdalena Slavkova à ce dossier.

83. Cf. Mančeva (Mila), "Trudova migracija na bălgarskite turci v Germanija. Sǎetnični migrantski mreži i kulturi », op. cit., p.6.

84. Voir Kabakchieva (Petya), "Temporary Migrants: Beyond Roles, Across Identities", in : Kiossev (Alexander), Kabakchieva (Petya), eds., Rules and Roles: Fluid Institutions, Hybrid Roles and Identities in East European Transformation Processes (1989 - 2005), Freiburg : LitVerlag (à paraître). 85. Voir Brubaker (Rogers), Feischmidt (Margit), Fox (Jon), Grancea (Liana), Nationalist Politics and Everyday Ethnicity in a Transylvanian Town, Princeton \& Oxford : Princeton University Press, 2006.

86. Cf. Veyne (Paul), Les Grecs ont-ils cru à leurs mythes ?, Paris : Le Seuil, 1983.

87. Voir Koselleck (Reinhardt), Le futur passé. Contributions à la sémantique des temps historiques,

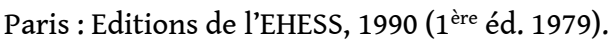

88. Voir Hartog (François), Régimes d'historicité. Présentisme et expériences du temps, Paris : Le Seuil, 2003.

89. Le terme gurbet, qui vient du turc, signifie "nostalgie »; en langue bulgare, il a reçu la connotation de « l'étranger » (au-delà des frontières du terroir d'origine).

90. Pour une démarche en ce sens, voir Waldinger (Roger), «Immigrant "Transnationalism » and the Presence of the Past », Los Angeles, UCLA, 2006, à l'adresse :http://www.sscnet.ucla.edu/ soc/faculty/waldinger/pdf/B10.pdf

91. Soultanova (Ralitsa), Les migrations multiples de la population bulgare, Université libre de Bruxelles, Groupe d'études sur l'ethnicité, le racisme, les migrations et l'exclusion (GERME), à l'adresse :

http://balkans.courriers.info/IMG/rtf/

Les_migrations_multiples_de_la_population_bulgare.rtf.

92. Voir Tapia (Stéphane de), "Migrations internationales et Anthropologie du voyage. La circulation des hommes et des biens dans le champ migratoire turc : itinéraires et impacts économiques", Paris: FASOPO, décembre 2006, à l'adresse: http://www.fasopo.org/ publications/anthropologievoyage_st_1206.pdf

93. Voir Fox (Jon), Identity Formation in Migration: The Case of Transylvanian Hungarian Guest Workers. Contribution à la Conférence Kokkalis, Harvard University, Cambridge, 2008, à l'adresse : http://www.hks.harvard.edu/kokkalis/GSW1/GSW1/05\%20Fox.pdf

94. Elle pourrait également dialoguer avec les travaux sur les Allemands de Transylvanie ayant pris le chemin de l'Allemagne dans le cadre des migrations dites « ethniques » ou des migrations "de retour». Voir Michalon (Bénédicte), « Migrations des Saxons de Roumanie en Allemagne. Mythe, interdépendance et altérité dans le 'retour' ", Thèse de doctorat dirigée par Michelle Guillon, Migrinter, décembre 2003.

\section{RÉSUMÉS}

L'objectif de cet article est triple: il est d'abord de mettre en perspective les processus migratoires intervenus ces vingt dernières années depuis la Bulgarie. L'accent est ici placé sur les profils migrants, les ressources de la mobilité et la manière dont les circulations ont contribué à une redéfinition des hiérarchies et imaginaires sociaux, ainsi que des rapports à l'espace et au temps. Sont également envisagées les incidences (moindres qu'on ne l'a parfois dit) de l'adhésion 
européenne sur les parcours migrants. En raison de la rareté relative des travaux consacrés aux migrations bulgares, ce texte vise en second lieu à retracer l'émergence, en Bulgarie comme à l'international, d'un champ de recherche sur les mobilités et de présenter au lecteur les principales problématiques à l'aune desquelles ces circulations ont été appréhendées. C'est par rapport à cette littérature qu'a été pensé le présent dossier dont la dernière partie de l'introduction restitue les principaux axes.

\section{INDEX}

Index géographique : Bulgarie

Mots-clés : Migrations, Immigration, Emigration, Mobilités

\section{AUTEUR}

\section{NADĖGE RAGARU}

Dr. Nadège Ragaruest chargée de recherche au CNRS (CERI-Sciences Po) et enseigne à Sciences Po-Paris. Diplômée de science politique, relations internationales et études est-européennes, elle a soutenu une thèse sur « Apprivoiser les transformations post-communistes en Bulgarie : la fabrique du politique (1989-2004)» (Sciences Po, 2005). Ses recherches portent sur les identifications et politiques de minorités en Bulgarie et en Macédoine, les transferts et circulations de normes internationales, ainsi que le communisme au quotidien dans la Bulgarie des années 1970-1980. Elle a récemment publié : « La rivière et les petits cailloux. Elargissement européen et européanisation en Europe centrale et orientale », in : F. Bafoil et T. Beichelt (dir.), L'européanisation d'Ouest en Est, Paris : L'Harmattan, 2008, p.241-283 ; « Les investissements symboliques du 'schisme' dans l'Eglise orthodoxe bulgare », in : A. Capelle-Pogăcean, P. Michel et E. Pace (dir.), Religion(s) et identité(s) en Europe. L'épreuve du pluriel, Paris : P. Sciences Po, 2008, p. 213-254; « Repenser la politisation des identités. Les engagements militants dans les Balkans d'aujourd'hui ", Revue d'études comparatives Est-Ouest, 38(4), décembre 2007, p.5-28 ; « Quelques remarques sur les échanges de services et l'appropriation de l'ordre politique en Bulgarie communiste », in : Sandrine Kott, Martine Mespoulet (dir.), Le post-communisme dans l'histoire, Bruxelles : PUB, 2006, p.51-62.

ragaru@ceri-sciences-po.org 